\title{
ON THE CHARACTER RING OF A FINITE GROUP
}

\author{
by
}

Cédric Bonnafé

\begin{abstract}
Let $G$ be a finite group and let $k$ be a sufficiently large finite field. Let $\mathcal{R}(G)$ denote the character ring of $G$ (i.e. the Grothendieck ring of the category of $\mathbb{C} G$-modules). We study the structure and the representations of the commutative algebra $k \otimes_{\mathbb{Z}} \mathcal{R}(G)$.
\end{abstract}

\section{Contents}

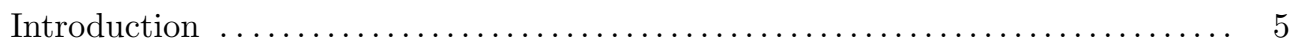

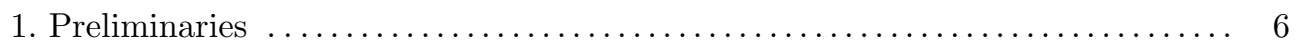

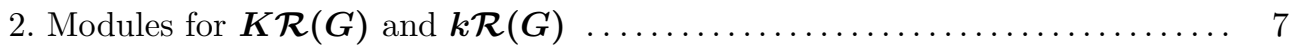

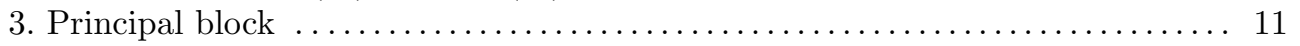

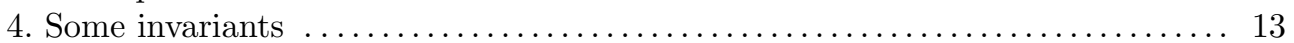

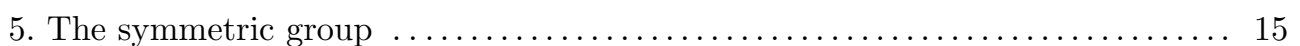

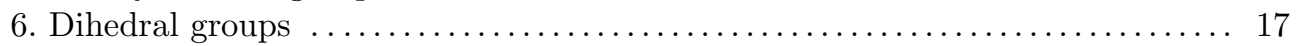

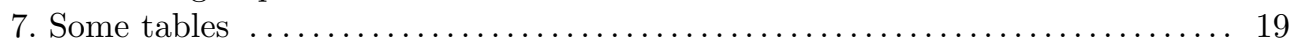

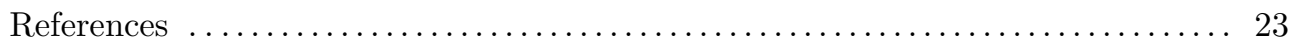

\section{Introduction}

Let $G$ be a finite group. We denote by $\mathcal{R}(G)$ the Grothendieck ring of the category of $\mathbb{C} G$ modules (it is usually called the character ring of $G$ ). It is a natural question to try to recover properties of $G$ from the knowledge of $\mathcal{R}(G)$. It is clear that two finite groups having the same character table have the same Grothendieck rings and it is a Theorem of Saksonov [S] that the converse also holds. So the problem is reduced to an intensively studied question in character theory: recover properties of the group through properties of its character table.

In this paper, we study the $k$-algebra $k \mathcal{R}(G)=k \otimes_{\mathbb{Z}} \mathcal{R}(G)$, where $k$ is a splitting field for $G$ of positive characteristic $p$. It is clear that the knowledge of $k \mathcal{R}(G)$ is a much weaker information than the knowledge of $\mathcal{R}(G)$. The aim of this paper is to gather results on the representation theory of the algebra $k \mathcal{R}(G)$ : although most of the results are certainy well-known, we have not found any general treatment of these questions. The blocks of $k \mathcal{R}(G)$ are local algebras which are parametrized by conjugacy classes of $p$-regular elements of $G$. So the simple $k \mathcal{R}(G)$-modules are parametrized by conjugacy classes of $p$-regular elements of $G$. Moreover, the dimension of the projective cover of the simple module associated to the conjugacy class of the $p$-regular element $g \in G$ is equal to the number of conjugacy classes of $p$-elements in the centralizer $C_{G}(g)$. We also prove that the radical of $k \mathcal{R}(G)$ is the kernel of the decomposition map $k \mathcal{R}(G) \rightarrow k \otimes_{\mathbb{Z}} \mathcal{R}(k G)$, where $\mathcal{R}(k G)$ is the Grothendieck ring of the category of $k G$-modules (i.e. the ring of virtual Brauer characters of $G)$. 
We prove that the block of $k \mathcal{R}(G)$ associated to the $p^{\prime}$-element $g$ is isomorphic to the block of $k \mathcal{R}\left(C_{G}(g)\right)$ associated to 1 (such a block is called the principal block). This shows that the study of blocks of $k \mathcal{R}(G)$ is reduced to the study of principal blocks. We also show that the principal block of $k \mathcal{R}(G)$ is isomorphic to the principal block of $k \mathcal{R}(H)$ whenever $H$ is a subgroup of $p^{\prime}$-index which controls the fusion of $p$-elements or whenever $H$ is the quotient of $G$ by a normal $p^{\prime}$-subgroup.

We also introduce several numerical invariants (Loewy length, dimension of Ext-groups) that are partly related to the structure of $G$. These numerical invariants are computed completely whenever $G$ is the symmetric group $\mathfrak{S}_{n}$ (this relies on previous work of the author: the descending Loewy series of $k \mathcal{R}\left(\mathfrak{S}_{n}\right)$ was entirely computed in [B]]) or $G$ is a dihedral group and $p=2$. We also provide tables for these invariants for small groups (alternating groups $\mathfrak{A}_{n}$ with $n \leqslant 12$, some small simple groups, groups $\operatorname{PSL}(2, q)$ with $q$ a prime power $\leqslant 27$, exceptional finite Coxeter groups).

Notation - Let $\mathcal{O}$ be a Dedekind domain of characteristic zero, let $\mathfrak{p}$ be a maximal ideal of $\mathcal{O}$, let $K$ be the fraction field of $\mathcal{O}$ and let $k=\mathcal{O} / \mathfrak{p}$. Let $\mathcal{O}_{\mathfrak{p}}$ be the localization of $\mathcal{O}$ at $\mathfrak{p}$ : then $k=\mathcal{O}_{\mathfrak{p}} / \mathfrak{p} \mathcal{O}_{\mathfrak{p}}$. If $x \in \mathcal{O}_{\mathfrak{p}}$, we denote by $\bar{x}$ its image in $\mathcal{O}_{\mathfrak{p}} / \mathfrak{p} \mathcal{O}_{\mathfrak{p}}=k$. Throughout this paper, we assume that $k$ has characteristic $p>0$ and that $K$ and $k$ are splitting fields for all the finite groups involved in this paper. If $n$ is a non-zero natural number, $n_{p^{\prime}}$ denotes the largest divisor of $n$ prime to $p$ and we set $n_{p}=n / n_{p^{\prime}}$.

If $F$ is a field and if $A$ is a finite dimensional $F$-algebra, we denote by $\mathcal{R}(A)$ its Grothendieck group. If $M$ is an $A$-module, the radical of $M$ is denoted by $\operatorname{Rad} M$ and the class of $M$ in $\mathcal{R}(A)$ is denoted by $[M]$. If $S$ is a simple $A$-module, we denote by $[M: S]$ the multiplicity of $S$ as a chief factor of a Jordan-Hölder series of $M$. The set of irreducible characters of $A$ is denoted by $\operatorname{Irr} A$.

We fix all along this paper a finite group $G$. For simplification, we set $\mathcal{R}(G)=\mathcal{R}(K G)$ and $\operatorname{Irr} G=\operatorname{Irr} K G$ (recall that $K$ is a splitting field for $G$ ). The abelian group $\mathcal{R}(G)$ is endowed with a structure of ring induced by the tensor product. If $\chi \in \mathcal{R}(G)$, we denote by $\chi^{*}$ its dual (as a class function on $G$, we have $\chi^{*}(g)=\chi\left(g^{-1}\right)$ for any $\left.g \in G\right)$. If $R$ is any commutative ring, we denote by $\operatorname{Class}_{R}(G)$ the space of class functions $G \rightarrow R$ and we set $R \mathcal{R}(G)=R \otimes_{\mathbb{Z}} \mathcal{R}(G)$. If $X$ is a subset of $G$, we denote by $1_{X}^{R}: G \rightarrow R$ the characteristic function of $X$. If $R$ is a subring of $K$, then we simply write $1_{X}=1_{X}^{R}$. Note that $1_{G}$ is the trivial character of $G$. If $f, f^{\prime} \in \operatorname{Class}_{K}(G)$, we set

$$
\left\langle f, f^{\prime}\right\rangle_{G}=\frac{1}{|G|} \sum_{g \in G} f\left(g^{-1}\right) f^{\prime}(g) .
$$

Then $\operatorname{Irr} G$ is an orthonormal basis of $\operatorname{Class}_{K}(G)$. We shall identify $\mathcal{R}(G)$ with the sub-ZZZ-module (or sub-ZZ-algebra) of $\operatorname{Class}_{K}(G)$ generated by $\operatorname{Irr} G$, and $K \mathcal{R}(G)$ with $\operatorname{Class}_{K}(G)$. If $f \in \mathcal{O}_{\mathfrak{p}} \mathcal{R}(G)$, we denote by $\bar{f}$ its image in $k \mathcal{R}(G)$.

If $g$ and $h$ are two elements of $G$, we write $g \sim h$ (or $g \sim_{G} h$ if we need to emphasize the group) if they are conjugate in $G$. We denote by $g_{p}$ (resp. $g_{p^{\prime}}$ ) the $p$-part (resp. the $p^{\prime}$-part) of $g$. If $X$ is a subset of $G$, we set $X_{p^{\prime}}=\left\{g_{p^{\prime}} \mid g \in X\right\}$ and $X_{p}=\left\{g_{p} \mid g \in X\right\}$. If moreover $X$ is closed under conjugacy, the set of conjugacy classes contained in $X$ is denoted by $X / \sim$. In this case, $1_{X}^{R} \in \operatorname{Class}_{R}(G)$. The centre of $G$ is denoted by $Z(G)$.

REMARK - We have recently discovered that some of the questions investigated in this paper were already studied by M. Deiml in his Ph.D. Thesis [D, Chapter 3]. More precisely, most of the results of our Section 2 were already proved by M. Deiml.

\section{Preliminaries}

1.A. Symmetrizing form. - Let

$$
\begin{aligned}
\tau_{G}: \mathcal{R}(G) & \longrightarrow \mathbb{Z} \\
\chi & \longmapsto\left\langle\chi, 1_{G}\right\rangle_{G}
\end{aligned}
$$


denote the canonical symmetrizing form on $\mathcal{R}(G)$. The dual basis of $\operatorname{Irr} G$ is $\left(\chi^{*}\right)_{\chi \in \operatorname{Irr} G}$. It is then readily seen that $(\mathcal{R}(G), \operatorname{Irr} G)$ is a based ring (in the sense of Lusztig [L, Page 236]).

If $R$ is any ring, we denote by $\tau_{G}^{R}: R \mathcal{R}(G) \rightarrow R$ the symmetrizing form $\operatorname{Id}_{R} \otimes_{\mathbb{Z}} \tau_{G}$.

1.B. Translation by the centre. - If $\chi \in \operatorname{Irr} G$, we denote by $\omega_{\chi}: Z(G) \rightarrow \mathcal{O}^{\times}$the linear character such that $\chi(z g)=\omega_{\chi}(z) \chi(g)$ for all $z \in Z(G)$ and $g \in G$. If $z \in Z(G)$, we denote by $t_{z}: K \mathcal{R}(G) \rightarrow K \mathcal{R}(G)$ the linear map defined by $\left(t_{z} f\right)(g)=f(z g)$ for all $f \in K \mathcal{R}(G)$ and $g \in G$. It is clear that $t_{z z^{\prime}}=t_{z} \circ t_{z^{\prime}}$ for all $z, z^{\prime} \in Z(G)$ and that $t_{z}$ is an automorphism of algebra. Moreover,

$$
t_{z} \chi=\omega_{\chi}(z) \chi
$$

for every $\chi \in \operatorname{Irr} G$. Therefore, $t_{z}$ is an isometry which stabilizes $\mathcal{O R}(G)$. If $R$ is a subring of $K$ such that $\mathcal{O} \subset R \subset K$, we still denote by $t_{z}: R \mathcal{R}(G) \rightarrow R \mathcal{R}(G)$ the restriction of $t_{z}$. Let $\bar{t}_{z}=\operatorname{Id}_{k} \otimes_{\mathcal{O}} t_{z}: k \mathcal{R}(G) \rightarrow k \mathcal{R}(G)$. This is again an automorphism of $k$-algebra. If $z$ is a $p$-element, then $\bar{t}_{z}=\operatorname{Id}_{k \mathcal{R}(G)}$.

1.C. Restriction. - If $\pi: H \rightarrow G$ is a morphism of groups, then the restriction through $\pi$ induces a morphism of rings $\operatorname{Res}_{\pi}: \mathcal{R}(G) \rightarrow \mathcal{R}(H)$. If $R$ is a subring of $K$, we still denote by $\operatorname{Res}_{\pi}: R \mathcal{R}(G) \rightarrow R \mathcal{R}(H)$ the morphism $\operatorname{Id}_{R} \otimes_{\mathbb{Z}} \operatorname{Res}_{\pi}$. We denote by $\overline{\operatorname{Res}}_{\pi}: k \mathcal{R}(G) \rightarrow k \mathcal{R}(H)$ the reduction modulo $\mathfrak{p}$ of $\operatorname{Res}_{\pi}: \mathcal{O R}(G) \rightarrow \mathcal{O R}(H)$. Recall that, if $H$ is a subgroup of $G$ and $\pi$ is the canonical injection, then $\operatorname{Res}_{\pi}$ is just $\operatorname{Res}_{H}^{G}$. In this case, $\overline{\operatorname{Res}}_{\pi}$ will be denoted by $\overline{\operatorname{Res}}_{H}^{G}$. Note the following fact:

$$
\text { If } \pi \text { is surjective, then } \overline{\operatorname{Res}}_{\pi} \text { is injective. }
$$

Proof of 1.1. - Indeed, if $\pi$ is surjective, then $\operatorname{Res}_{\pi}: \mathcal{R}(G) \rightarrow \mathcal{R}(H)$ is injective and its image is a direct summand of $\mathcal{R}(H)$.

1.D. Radical. - First, note that, since $k \mathcal{R}(G)$ is commutative, we have

$$
\operatorname{Rad} k \mathcal{R}(G) \text { is the ideal of nilpotent elements of } k \mathcal{R}(G) \text {. }
$$

So, if $\pi: H \rightarrow G$ is a morphism of finite groups, then

$$
\overline{\operatorname{Res}}_{\pi}(\operatorname{Rad} k \mathcal{R}(G)) \subset \operatorname{Rad} k \mathcal{R}(H) .
$$

The Loewy length of the algebra $k \mathcal{R}(G)$ is defined as the smallest natural number $n$ such that $(\operatorname{Rad} k \mathcal{R}(G))^{n}=0$. We denote it by $\ell_{p}(G)$. By 1.1 and 1.3 , we have:

$$
\text { If } \pi \text { is surjective, then } \ell_{p}(G) \leqslant \ell_{p}(H) \text {. }
$$

\section{Modules for $K \mathcal{R}(G)$ and $k \mathcal{R}(G)$}

2.A. Semisimplicity. - Recall that $K \mathcal{R}(G)$ is identified with the algebra of class functions on $G$. If $C \in G / \sim$ and $f \in K \mathcal{R}(G)$, we denote by $f(C)$ the constant value of $f$ on $C$. We now define $\operatorname{ev}_{C}: K \mathcal{R}(G) \rightarrow K, f \mapsto f(C)$. It is a morphism of $K$-algebras. In other words, it is an irreducible representation (or character) of $K \mathcal{R}(G)$. We denote by $\mathcal{D}_{C}$ the corresponding simple $K \mathcal{R}(G)$-module $\left(\operatorname{dim}_{K} \mathcal{D}_{C}=1\right.$ and an element $f \in K \mathcal{R}(G)$ acts on $\mathcal{D}_{C}$ by multiplication by $\left.\operatorname{ev}_{C}(f)=f(C)\right)$. Now, $1_{C}$ is a primitive idempotent of $K \mathcal{R}(G)$ and it is easily checked that

$$
K \mathcal{R}(G) 1_{C} \simeq \mathcal{D}_{C}
$$

Recall that

$$
1_{C}=\frac{|C|}{|G|} \sum_{\chi \in \operatorname{Irr} G} \chi\left(C^{-1}\right) \chi
$$


and

$$
\sum_{C \in G / \sim} 1_{C}=1_{G}
$$

Therefore:

Proposition 2.4. - We have:

(a) $\left(\mathcal{D}_{C}\right)_{C \in G / \sim}$ is a family of representatives of isomorphy classes of simple $K \mathcal{R}(G)$-modules.

(b) $\operatorname{Irr} K \mathcal{R}(G)=\left\{\mathrm{ev}_{C} \mid C \in G / \sim\right\}$.

(c) $K \mathcal{R}(G)$ is split semisimple.

We conclude this section by the computation of the Schur elements (see [GP, 7.2] for the definition) associated to each irreducible character of $K \mathcal{R}(G)$. Since

$$
\tau_{G}^{K}=\sum_{C \in G / \sim} \frac{|C|}{|G|} \mathrm{ev}_{C}
$$

we have by [GP, Theorem 7.2.6]:

Corollary 2.6. - Let $C \in G / \sim$. Then the Schur element associated with the irreducible character $\mathrm{ev}_{C}$ is $\frac{|G|}{|C|}$.

REMARK 2.7 - If $z \in Z(G)$, then $t_{z}$ induces an isomorphism of algebras $K \mathcal{R}(G) 1_{C} \simeq K \mathcal{R}(G) 1_{z^{-1} C}$.

Remark 2.8 - If $f \in K \mathcal{R}(G)$, then $f=\sum_{C \in G \mathcal{\sim}} f(C) 1_{C}$.

Example 2.9 - The map ev 1 will sometimes be denoted by deg, since it sends a character to its degree.

2.B. Decomposition map. - Let $d_{\mathfrak{p}}: \mathcal{R}(G) \rightarrow \mathcal{R}(k G)$ denote the decomposition map. If $R$ is any commutative ring, we denote by $d_{\mathfrak{p}}^{R}: R \mathcal{R}(G) \rightarrow R \mathcal{R}(k G)$ the induced map. Note that $\mathcal{R}(k G)$ is also a ring (for the multiplication given by tensor product) and that $d_{\mathfrak{p}}$ is a morphism of ring. Also, by [CR, Corollary 18.14],

$$
d_{\mathfrak{p}} \text { is surjective. }
$$

Since $\operatorname{Irr}(k G)$ is a linearly independent family of class functions $G \rightarrow k$ (see [CR, Theorem 17.4]), the map $\chi: k \mathcal{R}(k G) \rightarrow \operatorname{Class}_{k}(G)$ that sends the class of a $k G$-module to its character is (welldefined and) injective. This is a morphism of $k$-algebras.

Now, if $C$ is a conjugacy class of $p$-regular elements (i.e. $C \in G_{p^{\prime}} / \sim$ ), we define

$$
\mathcal{S}_{p^{\prime}}(C)=\left\{g \in G \mid g_{p^{\prime}} \in C\right\}
$$

(for instance, $\mathcal{S}_{p^{\prime}}(1)=G_{p}$ ). Then $\mathcal{S}_{p^{\prime}}(C)$ is called the $p^{\prime}$-section of $C$ : this is a union of conjugacy classes of $G$. Let $\operatorname{Class}_{k}^{p^{\prime}}(G)$ be the space of class functions $G \rightarrow k$ which are constant on $p^{\prime}$-sections. Then, by [CR, Lemma 17.8], $\operatorname{Irr}(k G) \subset \operatorname{Class}_{k}^{p^{\prime}}(G)$, so the image of $\chi$ is contained in $\operatorname{Class}_{k}^{p^{\prime}}(G)$. But, $\chi$ is injective, $|\operatorname{Irr}(k G)|=\left|G_{p^{\prime}} / \sim\right|\left(\right.$ see $\left[\mathbf{C R}\right.$, Corollary 17.11]) and $\operatorname{dim}_{k} \operatorname{Class}_{k}^{p^{\prime}}(G)=\mid G_{p^{\prime}} / \sim$ |. Therefore, we can identify, through $\chi$, the $k$-algebras $k \mathcal{R}(k G)$ and $\operatorname{Class}_{k}^{p^{\prime}}(G)$. In particular,

$$
k \mathcal{R}(k G) \text { is split semisimple. }
$$


2.C. Simple $\boldsymbol{k} \mathcal{R}(\boldsymbol{G})$-modules. - If $C \in G / \sim$, we still denote by ev $_{C}: \mathcal{O} \mathcal{R}(G) \rightarrow \mathcal{O}$ the restriction of $\operatorname{ev}_{C}$ and we denote by $\overline{\operatorname{ev}}_{C}: k \mathcal{R}(G) \rightarrow k$ the reduction modulo $\mathfrak{p}$ of ev . It is easily checked that $\overline{\mathrm{ev}}_{C}$ factorizes through the decomposition map $d_{\mathfrak{p}}$. Indeed, if $\operatorname{ev}_{C}^{k}: k \mathcal{R}(k G) \rightarrow$ $k$ denote the evaluation at $C$ (recall that $k \mathcal{R}(k G)$ is identified, via the map $\chi$ of the previous subsection, to $\left.\operatorname{Class}_{k}^{p^{\prime}}(G)\right)$, then

$$
\overline{\mathrm{ev}}_{C}=\mathrm{ev}_{C}^{k} \circ d_{\mathfrak{p}}^{k}
$$

Let $\overline{\mathcal{D}}_{C}$ be the corresponding simple $k \mathcal{R}(G)$-module. Let $\delta_{\mathfrak{p}}: \mathcal{R}(K \mathcal{R}(G)) \rightarrow \mathcal{R}(k \mathcal{R}(G))$ denote the decomposition map (see [GP, 7.4] for the definition). Then

$$
\delta_{\mathfrak{p}}\left[\mathcal{D}_{C}\right]=\left[\overline{\mathcal{D}}_{C}\right]
$$

The following facts are well-known:

Proposition 2.14. - Let $C, C^{\prime} \in G / \sim$. Then $\overline{\mathcal{D}}_{C} \simeq \overline{\mathcal{D}}_{C^{\prime}}$ if and only if $C_{p^{\prime}}=C_{p^{\prime}}^{\prime}$.

Proof. - The "if" part follows from the following classical fact [CR, Proposition 17.5 (ii) and (iv) and Lemma 17.8]: if $\chi \in \mathcal{R}(G)$ and if $g \in G$, then

$$
\chi(g) \equiv \chi\left(g_{p^{\prime}}\right) \quad \bmod \mathfrak{p} .
$$

The "only if" part follows from 2.12 and from the surjectivity of the decomposition map $d_{\mathfrak{p}}$.

Corollary 2.15. - We have:

(a) $\left(\overline{\mathcal{D}}_{C}\right)_{C \in G_{p^{\prime}} / \sim}$ is a family of representatives of isomorphy classes of simple $k \mathcal{R}(G)$-modules.

(b) $\operatorname{Irr} k \mathcal{R}(G)=\left\{\overline{\operatorname{ev}}_{C} \mid C \in G_{p^{\prime}} / \sim\right\}$.

(c) $\operatorname{Rad} k \mathcal{R}(G)=\operatorname{Ker} d_{\mathfrak{p}}^{k}$.

(d) $k \mathcal{R}(G)$ is split.

Proof. - (a) follows from 2.13 and from the fact that the isomorphy class of any simple $k \mathcal{R}(G)$ modules must occur in some $\delta_{\mathfrak{p}}[S]$, where $S$ is a simple $K \mathcal{R}(G)$-module. (b) follows from (a). (c) and (d) follow from (a), (b), 2.12 and 2.11 .

Corollary 2.16. $-\operatorname{dim}_{k} \operatorname{Rad}(k \mathcal{R}(G))=|G / \sim|-\left|G_{p^{\prime}} / \sim\right|$

Corollary 2.17. - $k \mathcal{R}(G)$ is semisimple if and only if $p$ does not divide $|G|$.

EXAMPLE 2.18 - Since $e_{1}$ is also denoted by deg, we shall sometimes denote by $\overline{\operatorname{deg}}$ the morphism $\overline{\mathrm{ev}}_{1}$. If $G$ is a $p$-group, then Corollary 2.15 shows that $\operatorname{Rad} k \mathcal{R}(G)=\operatorname{Ker}(\overline{\operatorname{deg}})$. In this case, if $1, \lambda_{1}, \ldots, \lambda_{r}$ denote the linear characters of $G$ and $\chi_{1}, \ldots, \chi_{s}$ denote the non-linear irreducible characters of $G$, then $\left(\bar{\lambda}_{1}-1, \ldots, \bar{\lambda}_{r}-1, \bar{\chi}_{1}, \bar{\chi}_{s}\right)$ is a $k$-basis of $\operatorname{Rad} k \mathcal{R}(G)$.

2.D. Projective modules. - We now fix a conjugacy class $C$ of $p$-regular elements (i.e. $C \in$ $\left.G_{p^{\prime}} / \sim\right)$. Let

$$
e_{C}=1_{\mathcal{S}_{p^{\prime}}(C)}=\sum_{D \in \mathcal{S}_{p^{\prime}}(C) / \sim} 1_{D} .
$$

If necessary, $e_{C}$ will be denoted by $e_{C}^{G}$. If $H$ is a subgroup of $G$, then

$$
\operatorname{Res}_{H}^{G} e_{C}^{G}=\sum_{D \in(C \cap H) / \sim_{H}} e_{D}^{H} .
$$

Proposition 2.20. - Let $C \in G_{p^{\prime}} / \sim$. Then $e_{C} \in \mathcal{O}_{\mathfrak{p}} \mathcal{R}(G)$. 
Proof. - Using Brauer's Theorem, we only need to prove that $\operatorname{Res}_{N}^{G} e_{C}^{G} \in \mathcal{O}_{\mathfrak{p}} \mathcal{R}(N)$ for every nilpotent subgroup $N$ of $G$. By 2.19, this amounts to prove the lemma whenever $G$ is nilpotent. So we assume that $G$ is nilpotent. Then $G=G_{p^{\prime}} \times G_{p}$, and $G_{p}$ and $G_{p^{\prime}}$ are subgroups of $G$. Moreover, $C \subset G_{p^{\prime}}$ and $\mathcal{S}_{p^{\prime}}(G)=C \times G_{p}$. If we identify $K \mathcal{R}(G)$ and $K \mathcal{R}\left(G_{p^{\prime}}\right) \otimes_{K} K \mathcal{R}\left(G_{p}\right)$, we have $e_{C}^{G}=1_{C}^{G_{p^{\prime}}} \otimes_{\mathcal{O}_{\mathfrak{p}}} e_{1}^{G_{p}}$. But, by 2.2, we have that $e_{C}^{G_{p^{\prime}}} \in \mathcal{O}_{\mathfrak{p}} \mathcal{R}\left(G_{p^{\prime}}\right)$. On the other hand, $e_{1}^{G_{p}}=1_{G_{p}} \in \mathcal{R}\left(G_{p}\right)$. The proof of the lemma is complete.

Corollary 2.21. - Let $C \in G_{p^{\prime}} / \sim$. Then $e_{C}$ is a primitive idempotent of $\mathcal{O}_{\mathfrak{p}} \mathcal{R}(G)$.

Proof. - By Proposition 2.15 (a), the number of primitive idempotents of $k \mathcal{R}(G)$ is $\left|G_{p^{\prime}} / \sim\right|$. So the number of primitive idempotents of $\hat{\mathcal{O}}_{\mathfrak{p}} \mathcal{R}(G)$ is also $\left|G_{p^{\prime}} / \sim\right|$ (here, $\hat{\mathcal{O}}_{\mathfrak{p}}$ denotes the completion

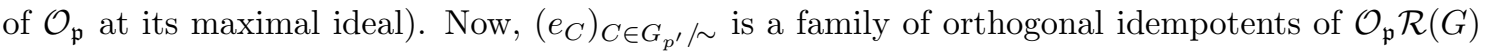
(see Proposition 2.20) and $1_{G}=\sum_{C \in G_{p^{\prime}} / e_{C}}$. The proof of the lemma is complete.

Let $\bar{e}_{C} \in k \mathcal{R}(G)$ denote the reduction modulo $\mathfrak{p} \mathcal{O}_{\mathfrak{p}}$ of $e_{C}$. Then it follows from 2.12 that

$$
d_{\mathfrak{p}}^{k} \bar{e}_{C}=1_{\mathcal{S}_{p^{\prime}}(C)}^{k} \in k \mathcal{R}(k G) \simeq \operatorname{Class}_{k}^{p^{\prime}}(G) .
$$

Let $\mathcal{P}_{C}=\mathcal{O}_{\mathfrak{p}} \mathcal{R}(G) e_{C}$ and $\overline{\mathcal{P}}_{C}=k \mathcal{R}(G) \bar{e}_{C}$ : they are indecomposable projective modules for $\mathcal{O}_{\mathfrak{p}} \mathcal{R}(G)$ and $k \mathcal{R}(G)$ respectively. Then

$$
\begin{aligned}
& \mathcal{O}_{\mathfrak{p}} \mathcal{R}(G)=\underset{C \in G_{p^{\prime}} / \sim}{\oplus} \mathcal{P}_{C} \\
& k \mathcal{R}(G)=\underset{C \in G_{p^{\prime}} / \sim}{\oplus} \overline{\mathcal{P}}_{C} .
\end{aligned}
$$

and

Note also that

$$
\operatorname{dim}_{k} k \mathcal{R}(G) \bar{e}_{C}=\operatorname{rank}_{\mathcal{O}_{\mathfrak{p}}} \mathcal{O}_{\mathfrak{p}} \mathcal{R}(G) e_{C}=\left|\mathcal{S}_{p^{\prime}}(G) / \sim\right|
$$

Proposition 2.24. - Let $C$ and $C^{\prime}$ be two conjugacy classes of $p^{\prime}$-regular elements of $G$. Then:

(a) $\left[\overline{\mathcal{P}}_{C}: \overline{\mathcal{D}}_{C^{\prime}}\right]=\left\{\begin{array}{ll}\left|\mathcal{S}_{p^{\prime}}(C) / \sim\right| & \text { if } C=C^{\prime}, \\ 0 & \text { otherwise. }\end{array}\right.$.

(b) $\overline{\mathcal{P}}_{C} / \operatorname{Rad} \overline{\mathcal{P}}_{C} \simeq \overline{\mathcal{D}}_{C}$.

Proof. - Let us first prove (a). By definition of $e_{C}$, we have

$$
\left[K \otimes_{\mathcal{O}_{\mathfrak{p}}} \mathcal{P}_{C}\right]=\sum_{D \in \mathcal{S}_{p^{\prime}}(G) / \sim}\left[\mathcal{D}_{D}\right] .
$$

Also, by definition of the decomposition map $\delta_{\mathfrak{p}}: \mathcal{R}(K \mathcal{R}(G)) \rightarrow \mathcal{R}(k \mathcal{R}(G))$, we have

$$
\delta_{\mathfrak{p}}\left[K \otimes_{\mathcal{O}_{\mathfrak{p}}} \mathcal{P}_{C}\right]=\left[\overline{\mathcal{P}}_{C}\right] .
$$

So the result follows from these observations and from 2.13. Now, (b) follows easily from (a).

2.E. More on the radical. - Let $\operatorname{Rad}_{p}(G)$ denote the set of functions $f \in \mathcal{O}_{\mathfrak{p}} \mathcal{R}(G)$ whose restriction to $G_{p^{\prime}}$ is zero. Note that $\operatorname{Rad}_{p}(G)$ is a direct summand of the $\mathcal{O}_{\mathfrak{p}}$-module $\mathcal{O}_{\mathfrak{p}} \mathcal{R}(G)$. So, $k \operatorname{Rad}_{p}(G)=k \otimes_{\mathcal{O}_{\mathfrak{p}}} \operatorname{Rad}_{p}(G)$ is a sub- $k$-vector space of $k \mathcal{R}(G)$.

Proposition 2.25. - We have:

(a) $\operatorname{dim}_{k} k \operatorname{Rad}_{p}(G)=|G / \sim|-\left|G_{p^{\prime}} / \sim\right|$.

(b) $k \operatorname{Rad}_{p}(G)$ is the radical of $k \mathcal{R}(G)$.

Proof. - (a) is clear. (b) follows from 2.12 and from Corollary 2.15.

Corollary 2.26. - Let e be the number such that $p^{e}$ is the exponent of a Sylow p-subgroup of G. If $f \in \operatorname{Rad} k \mathcal{R}(G)$, then $f^{p^{e}}=0$. 
Proof. - Let $e=e_{p}(G)$. If $f \in K \mathcal{R}(G)$ and if $n \geqslant 1$, we denote by $f^{(n)}: G \rightarrow K, g \mapsto f\left(g^{n}\right)$. Then the map $K \mathcal{R}(G) \rightarrow K \mathcal{R}(G), f \mapsto f^{(n)}$ is a morphism of $K$-algebras. Moreover (see for instance [CR, Corollary 12.10]), we have

$$
\text { If } f \in \mathcal{R}(G) \text {, then } f^{(n)} \in \mathcal{R}(G) \text {. }
$$

Therefore, it induces a morphism of $k$-algebras $\theta_{n}: k \mathcal{R}(G) \rightarrow k \mathcal{R}(G)$. Now, let $F: k \mathcal{R}(G) \rightarrow$ $k \mathcal{R}(G), \lambda \otimes_{\mathbb{Z}} f \mapsto \lambda^{p} \otimes_{\mathbb{Z}} f$. Then $F$ is an injective endomorphism of the ring $k \mathcal{R}(G)$. Moreover (see for instance [I, Problem 4.7]), we have

$$
F \circ \theta_{p}(f)=f^{p}
$$

for every $f \in k \mathcal{R}(G)$. Since $F$ and $\theta_{p}$ commute, we have $F^{e} \circ \theta_{p^{e}}(f)=f^{p^{e}}$ for every $f \in k \mathcal{R}(G)$. Therefore, if $\chi \in \operatorname{Rad}_{p}(G)$, we have

$$
\bar{\chi}^{p^{e}}=F^{e}\left(\overline{\chi^{\left(p^{e}\right)}}\right) .
$$

But, by hypothesis, $g^{p^{e}} \in G_{p^{\prime}}$ for every $g \in G$. So, if $f \in \operatorname{Rad}_{p}(G)$, then $f^{\left(p^{e}\right)}=0$. Therefore, $\bar{f}^{p^{e}}=0$. The corollary follows from this observation and from Proposition 2.25.

\section{Principal block}

If $C \in G_{p^{\prime}} / \sim$, we denote by $\mathcal{R}_{\mathfrak{p}}(G, C)$ the $\mathcal{O}_{\mathfrak{p}}$-algebra $\mathcal{O}_{\mathfrak{p}} \mathcal{R}(G) e_{C}$. As an $\mathcal{O}_{\mathfrak{p}} \mathcal{R}(G)$-module, this is just $\mathcal{P}_{C}$, but we want to study here its structure as a ring, so that is why we use a different notation. If $R$ is a commutative $\mathcal{O}_{\mathfrak{p}}$-algebra, we set $R \mathcal{R}_{\mathfrak{p}}(G, C)=R \otimes_{\mathcal{O}_{\mathfrak{p}}} \mathcal{R}_{\mathfrak{p}}(G, C)$. For instance, $k \mathcal{R}_{\mathfrak{p}}(G, C)=k \mathcal{R}(G) \bar{e}_{C}$, and $K \mathcal{R}_{\mathfrak{p}}(G, C)$ can be identified with the algebra of class functions on $\mathcal{S}_{p^{\prime}}(C)$.

The algebra $\mathcal{R}_{\mathfrak{p}}(G, 1)$ (resp. $\quad k \mathcal{R}_{\mathfrak{p}}(G, 1)$ ) will be called the principal block of $\mathcal{O}_{\mathfrak{p}} \mathcal{R}(G)$ (resp. $k \mathcal{R}(G))$. The aim of this section is to construct an isomorphism $\mathcal{R}_{\mathfrak{p}}(G, C) \simeq \mathcal{R}_{\mathfrak{p}}\left(C_{G}(g), 1\right)$, where $g$ is any element of $C$. We also emphasize the functorial properties of the principal block.

REMARK 3.1 - If $C \in G_{p^{\prime}} / \sim$ and if $z \in Z(G)$, then $t_{z}$ induces an isomorphism of algebras $\mathcal{R}_{\mathfrak{p}}(G, C) \simeq \mathcal{R}_{\mathfrak{p}}\left(G, z_{p^{\prime}}^{-1} C\right)$ (see Remark 2.7). Consequently, $\bar{t}_{z}$ induces an isomorphism of algebras $k \mathcal{R}_{\mathfrak{p}}(G, C) \simeq k \mathcal{R}_{\mathfrak{p}}\left(G, z^{-1} C\right)$.

3.A. Centralizers. - Let $C \in G_{p^{\prime}} / \sim_{G}$. Let $\operatorname{proj}_{C}^{G}: K \mathcal{R}(G) \rightarrow K \mathcal{R}_{\mathfrak{p}}(G, C), x \mapsto x e_{C}$ denote the canonical projection. We still denote by $\operatorname{proj}_{C}^{G}: \mathcal{O}_{\mathfrak{p}} \mathcal{R}(G) \rightarrow \mathcal{R}_{\mathfrak{p}}(G, C)$, the restriction of $\operatorname{proj}_{C}^{G}$ and we denote by $\overline{\operatorname{proj}}_{C}^{G}: k \mathcal{R}(G) \rightarrow k \mathcal{R}_{\mathfrak{p}}(G, C)$ its reduction modulo $\mathfrak{p} \mathcal{O}_{\mathfrak{p}}$.

Let us now fix $g \in C$. It is well-known (and easy) that the map $C_{G}(g)_{p} / \sim_{C_{G}(g)} \rightarrow \mathcal{S}_{p^{\prime}}(C) / \sim_{G}$ that sends the $C_{G}(g)$-conjugacy class $D \in C_{G}(g)_{p} / \sim_{C_{G}(g)}$ to the $G$-conjugacy class containing $g D$ is bijective. In particular,

$$
\left|\mathcal{S}_{p^{\prime}}(C) / \sim_{G}\right|=\left|C_{G}(g)_{p} / \sim_{C_{G}(g)}\right| .
$$

Now, let $d_{g}^{G}: K \mathcal{R}(G) \rightarrow K \mathcal{R}\left(C_{G}(g)\right)$ be the map defined by:

$$
\left(d_{g}^{G} f\right)(h)= \begin{cases}f(g h) & \text { if } h \in C_{G}(g)_{p}, \\ 0 & \text { otherwise },\end{cases}
$$

for all $f \in K \mathcal{R}(G)$ and $h \in C_{G}(g)$. Then $d_{g}^{G} f \in K \mathcal{R}_{\mathfrak{p}}\left(C_{G}(g), 1\right)$. It must be noticed that

$$
d_{g}^{G}=\operatorname{proj}_{1}^{C_{G}(g)} \circ t_{g}^{C_{G}(g)} \circ \operatorname{Res}_{C_{G}(g)}^{G}=t_{g}^{C_{G}(g)} \circ \operatorname{proj}_{g}^{C_{G}(g)} \circ \operatorname{Res}_{C_{G}(g)}^{G} .
$$

In particular, $d_{g}^{G}$ sends $\mathcal{O}_{\mathfrak{p}} \mathcal{R}(G)$ to $\mathcal{R}_{\mathfrak{p}}\left(C_{G}(g), 1\right)$. We denote by $\operatorname{res}_{g}: \mathcal{R}_{\mathfrak{p}}(G, C) \rightarrow \mathcal{R}_{\mathfrak{p}}\left(C_{G}(g), 1\right)$ the restriction of $d_{g}^{G}$ to $\mathcal{R}_{\mathfrak{p}}(G, C)$. Let $\operatorname{ind}_{g}: K \mathcal{R}_{\mathfrak{p}}\left(C_{G}(g), 1\right) \rightarrow K \mathcal{R}_{\mathfrak{p}}(G, C)$ be the map defined by

$$
\operatorname{ind}_{g} f=\operatorname{Ind}_{C_{G}(g)}^{G}\left(t_{g^{-1}}^{C_{G}(g)} f\right)
$$


for every $f \in K \mathcal{R}_{\mathfrak{p}}\left(C_{G}(g), 1\right)$. It is clear that $\operatorname{ind}_{g} f \in \mathcal{R}_{\mathfrak{p}}(G, C)$ if $f \in \mathcal{R}_{\mathfrak{p}}\left(C_{G}(g), 1\right)$. Thus we have defined two maps

and

$$
\operatorname{res}_{g}: \mathcal{R}_{\mathfrak{p}}(G, C) \rightarrow \mathcal{R}_{\mathfrak{p}}\left(C_{G}(g), 1\right)
$$

$$
\operatorname{ind}_{g}: \mathcal{R}_{\mathfrak{p}}\left(C_{G}(g), 1\right) \rightarrow \mathcal{R}_{\mathfrak{p}}(G, C) .
$$

We have:

Theorem 3.4. - If $g \in G_{p^{\prime}}$, then $\operatorname{res}_{g}$ and ind $_{g}$ are isomorphisms of $\mathcal{O}_{\mathfrak{p}}$-algebras inverse to each other.

Proof. - We first want to prove that $\operatorname{res}_{g} \circ \operatorname{ind}_{g}$ is the identity morphism. Let $f \in K \mathcal{R}_{\mathfrak{p}}\left(C_{G}(g), 1\right)$. Let $f^{\prime}=t_{g^{-1}} f$ and let $x \in C_{G}(g)_{p}$. We just need to prove that

$$
\left(\operatorname{Ind}_{C_{G}(g)}^{G} f^{\prime}\right)(g x)=f^{\prime}(g x) .
$$

But, by definition,

$$
\left(\operatorname{Ind}_{C_{G}(g)}^{G} f^{\prime}\right)(g x)=\sum_{\substack{h \in\left[G / C_{G}(g)\right] \\ h(g x) h^{-1} \in C_{G}(g)}} f^{\prime}\left(h(g x) h^{-1}\right) .
$$

Here, $\left[G / C_{G}(g)\right]$ denotes a set of representatives of $G / C_{G}(g)$. Since $f^{\prime}$ has support in $g C_{G}(g)_{p}$, we have $f\left(h(g x) h^{-1}\right) \neq 0$ only if the $p^{\prime}$-part of $h(g x) h^{-1}$ is equal to $g$, which happens if and only if $h \in C_{G}(g)$. This shows (?).

The fact that ind ${ }_{g} \circ \mathrm{res}_{g}$ is the identity can be proved similarly, or can be proved by using a trivial dimension argument. Since $\operatorname{res}_{g}$ is a morphism of algebras, we get that ind $\mathrm{d}_{g}$ is also a morphism of algebras.

3.B. Subgroups of index prime to $\boldsymbol{p}$. - If $H$ is a subgroup of $G$, then the restriction map $\operatorname{Res}_{H}^{G}$ sends $\mathcal{R}_{\mathfrak{p}}(G, 1)$ to $\mathcal{R}_{\mathfrak{p}}(H, 1)$ (indeed, by 2.19 , we have $\operatorname{Res}_{H}^{G} e_{1}^{G}=e_{1}^{H}$ ).

Theorem 3.5. - If $H$ is a subgroup of $G$ of index prime to $p$, then $\operatorname{Res}_{H}^{G}: \mathcal{R}_{\mathfrak{p}}(G, 1) \rightarrow \mathcal{R}_{\mathfrak{p}}(H, 1)$ is a split injection of $\mathcal{O}_{\mathfrak{p}}$-modules.

Proof. - Let us first prove that $\operatorname{Res}_{H}^{G}$ is injective. For this, we only need to prove that the map $\operatorname{Res}_{H}^{G}: K \mathcal{R}_{\mathfrak{p}}(G, 1) \rightarrow K \mathcal{R}_{\mathfrak{p}}(H, 1)$. But $K \mathcal{R}_{\mathfrak{p}}(G, 1)$ is the space of functions whose support is contained in $G_{p}$. Since the index of $H$ is prime to $p$, every conjugacy class of $p$-elements of $G$ meets $H$. This shows that $\operatorname{Res}_{H}^{G}$ is injective.

In order to prove that it is a split injection, we only need to prove that the $\mathcal{O}_{\mathfrak{p}}$-module $\mathcal{R}_{\mathfrak{p}}(H, 1) / \operatorname{Res}_{H}^{G}\left(\mathcal{R}_{\mathfrak{p}}(G, 1)\right)$ is torsion-free. Let $\pi$ be a generator of the ideal $\mathfrak{p} \mathcal{O}_{\mathfrak{p}}$. Let $\gamma \in \mathcal{R}_{\mathfrak{p}}(G, 1)$ and $\eta \in \mathcal{R}_{\mathfrak{p}}(H, 1)$ be such that $\pi \eta=\operatorname{Res}_{H}^{G} \gamma$. We only need to prove that $\gamma / \pi \in \mathcal{R}_{\mathfrak{p}}(G, 1)$. By Brauer's Theorem, it is sufficient to show that, for any nilpotent subgroup $N$ of $G$, we have $\operatorname{Res}_{N}^{G} \gamma \in \pi \mathcal{O}_{\mathfrak{p}} \mathcal{R}(N)$.

So let $N$ be a nilpotent subgroup. We have $N=N_{p} \times N_{p^{\prime}}$ and, since the index of $H$ in $G$ is prime to $p$, we may assume that $N_{p} \subset H$. Since $\operatorname{Res}_{N}^{G} \psi \in \mathcal{R}_{\mathfrak{p}}(N, 1)=\mathcal{O}_{\mathfrak{p}} \mathcal{R}\left(N_{p}\right) \otimes_{\mathcal{O}_{\mathfrak{p}}} e_{1}^{N_{p^{\prime}}}$, we have

$$
\begin{aligned}
\operatorname{Res}_{N}^{G} \gamma & =\left(\operatorname{Res}_{N_{p}}^{G} \gamma\right) \otimes_{\mathcal{O}_{\mathfrak{p}}} e_{1}^{N_{p^{\prime}}} \\
& =\left(\pi \operatorname{Res}_{N_{p}}^{H} \eta\right) \otimes_{\mathcal{O}_{\mathfrak{p}}} e_{1}^{N_{p^{\prime}}} \in \pi \mathcal{O}_{\mathfrak{p}} \mathcal{R}(N),
\end{aligned}
$$

as expected.

Corollary 3.6. - If $H$ is a subgroup of $G$ of index prime to $p$, then the map $\overline{\operatorname{Res}_{H}}$ : $k \mathcal{R}_{\mathfrak{p}}(G, 1) \rightarrow$ $k \mathcal{R}_{\mathfrak{p}}(H, 1)$ is an injective morphism of $k$-algebras.

Corollary 3.7. - If $H$ is a subgroup of $G$ of index prime to $p$ which controls the fusion of $p$ elements, then $\operatorname{Res}_{H}^{G}: \mathcal{R}_{\mathfrak{p}}(G, 1) \rightarrow \mathcal{R}_{\mathfrak{p}}(H, 1)$ is an isomorphism of $\mathcal{O}_{\mathfrak{p}}$-algebras. 
Proof. - In this case, $\operatorname{dim}_{K} K \mathcal{R}_{\mathfrak{p}}(G, 1)=\operatorname{dim}_{K} K \mathcal{R}_{\mathfrak{p}}(H, 1)$, so the result follows from Corollary 3.6 .

ExAmple 3.8 - Let $P$ be a Sylow $p$-subgroup of $G$ and assume in this example that $P$ is abelian. Then $N_{G}(P)$ controls the fusion of $p$-elements. It then follows from Corollary 3.7 that the restriction from $G$ to $N_{G}(P)$ induces isomorphisms of algebras $\mathcal{R}_{\mathfrak{p}}(G, 1) \simeq \mathcal{R}_{\mathfrak{p}}\left(N_{G}(P), 1\right)$ and $k \mathcal{R}_{\mathfrak{p}}(G, 1) \simeq$ $k \mathcal{R}_{\mathfrak{p}}\left(N_{G}(P), 1\right)$. In particular, $\ell_{p}(G, 1)=\ell_{p}\left(N_{G}(P), 1\right)$.

Example 3.9 - Let $N$ be a $p^{\prime}$-group, let $H$ be a group acting on $N$ and let $G=H \ltimes N$. Then $H$ is of index prime to $p$ and controls the fusion of $p$-elements of $G$. So $\operatorname{Res}_{H}^{G}$ induces isomorphisms of algebras $\mathcal{R}_{\mathfrak{p}}(G, 1) \simeq \mathcal{R}_{\mathfrak{p}}(H, 1)$ and $k \mathcal{R}_{\mathfrak{p}}(G, 1) \simeq k \mathcal{R}_{\mathfrak{p}}(H, 1)$. In particular, $\ell_{p}(G, 1)=\ell_{p}(H, 1)$.

3.C. Quotient by a normal $\boldsymbol{p}^{\prime}$-subgroup. - Let $N$ be a normal subgroup of $G$. Let $\pi: G \rightarrow$ $G / N$ denote the canonical morphism. Then the morphism of algebras $\operatorname{Res}_{\pi}: \mathcal{R}_{\mathfrak{p}}(G / N) \rightarrow \mathcal{R}_{\mathfrak{p}}(G)$ induces a morphism of algebras $\operatorname{Res}_{\pi}^{(1)}: \mathcal{R}_{\mathfrak{p}}(G / N, 1) \rightarrow \mathcal{R}_{\mathfrak{p}}(G, 1), f \mapsto\left(\operatorname{Res}_{\pi} f\right) e_{1}^{G}$. Note that $\operatorname{Res}_{\pi}^{(1)} e_{1}^{G / N}=e_{1}^{G}$. We denote by $\overline{\operatorname{Res}}_{\pi}^{(1)}: k \mathcal{R}_{\mathfrak{p}}(G / N, 1) \rightarrow k \mathcal{R}_{\mathfrak{p}}(G, 1)$ the morphism induced by $\operatorname{Res}_{\pi}^{(1)}$. Then:

Theorem 3.10. - With the above notation, we have:

(a) $\operatorname{Res}_{\pi}^{(1)}$ is a split injection of $\mathcal{O}_{\mathfrak{p}}$-modules.

(b) If $N$ is prime to $p$, then $\operatorname{Res}_{\pi}^{(1)}$ is an isomorphism.

Proof. - (a) The injectivity of $\operatorname{Res}_{\pi}^{(1)}$ follows from the fact that $(G / N)_{p}=G G_{p} N / N$. Now, let $I$ denote the image of $\operatorname{Res}_{\pi}^{(1)}$. Since $\operatorname{Res}_{\pi}\left(\mathcal{O}_{\mathfrak{p}} \mathcal{R}(G / N)\right)$ is a direct summand of $\mathcal{O}_{\mathfrak{p}} \mathcal{R}(G)$, we get that $\operatorname{Res}_{\pi}\left(\mathcal{R}_{\mathfrak{p}}(G / N, 1)\right)=\left(\operatorname{Res}_{\pi}^{(1)} e_{1}^{G / N}\right) \operatorname{Res}_{\pi}\left(\mathcal{O}_{\mathfrak{p}} \mathcal{R}(G / N)\right)$ is a direct summand of $\mathcal{O}_{\mathfrak{p}} \mathcal{R}(G)$. Since $I=e_{1}^{G} \operatorname{Res}_{\pi}\left(\mathcal{R}_{\mathfrak{p}}(G / N, 1)\right)$ and $e_{1}^{G}=e_{1}^{G} \operatorname{Res}_{\pi}\left(e_{1}^{G / N}\right)$, we get that $I=e_{1}^{G} \operatorname{Res}_{\pi}\left(\mathcal{O}_{\mathfrak{p}} \mathcal{R}(G / N)\right)$ is a direct summand of $\mathcal{O}_{\mathfrak{p}} \mathcal{R}(G)$, as desired.

(b) now follows from (a) and from the fact that the map $\pi$ induces a bijection between $G_{p} / \sim_{G}$ and $(G / N)_{g} / \sim_{G / N}$ whenever $N$ is a normal $p^{\prime}$-subgroup.

\section{Some invariants}

We introduce in this section some numerical invariants of the $k$-algebra $k \mathcal{R}(G)$ (more precisely, of the algebras $\left.k \mathcal{R}_{\mathfrak{p}}(G, C)\right)$ : Loewy length, dimension of the Ext-groups.

4.A. Loewy length. - If $C \in G_{p^{\prime}} / \sim$, we denote by $\ell_{p}(G, C)$ the Loewy length of the $k$-algebra $k \mathcal{R}_{\mathfrak{p}}(G, C)$. Then, by definition, we have

$$
\ell_{p}(G)=\max _{C \in G_{p^{\prime}} / \sim} \ell_{p}(G, C) .
$$

On the other hand, by Theorem 3.4, we have

$$
\text { If } C \in G_{p^{\prime}} / \sim \text { and if } g \in C \text {, then } \ell_{p}(G, C)=\ell_{p}\left(C_{G}(g), 1\right) \text {. }
$$

The following bound on the Loewy length of $k \mathcal{R}(G)$ is obtained immediately from 2.23 and 3.2:

$$
\ell_{p}(G) \leqslant \max _{C \in G_{p^{\prime}} / \sim}\left|\mathcal{S}_{p^{\prime}}(C) / \sim\right|=\max _{g \in G_{p^{\prime}}}\left|C_{G}(g)_{p} / \sim_{C_{G}(g)}\right| .
$$

We set $S_{p}(G)=\max _{C \in G_{p^{\prime}} / \sim}\left|\mathcal{S}_{p^{\prime}}(C) / \sim\right|$.

ExAmPLE 4.4 - The inequality 4.3 might be strict. Indeed, if $G=\mathbb{Z} / 2 \mathbb{Z} \times \mathbb{Z} / 2 \mathbb{Z}$, then $\ell_{2}(G)=$ $3<4=S_{2}(G)$. 
EXAMPLE 4.5 - If $S_{p}(G)=2$, then $\ell_{p}(G)=2$. Indeed, in this case, we have that $p$ divides $|G|$, so $k \mathcal{R}(G)$ is not semisimple by Corollary 2.17 , so $\ell_{p}(G) \geqslant 2$. The result then follows from 4.3.

4.B. Ext-groups. - If $i \geqslant 0$ and if $C \in G_{p^{\prime}} / \sim$, we set

$$
\operatorname{ext}_{p}^{i}(G, C)=\operatorname{dim}_{\mathbb{F}_{p}} \operatorname{Ext}_{k \mathcal{R}(G)}^{i}\left(\overline{\mathcal{D}}_{C}, \overline{\mathcal{D}}_{C}\right) .
$$

Note that $\operatorname{ext}_{p}^{i}(G, C)=\operatorname{dim}_{\mathbb{F}_{p}} \operatorname{Ext}_{k \mathcal{R}_{\mathfrak{p}}(G, C)}^{i}\left(\overline{\mathcal{D}}_{C}, \overline{\mathcal{D}}_{C}\right)$. So, if $g \in C$, it follows from Theorem 3.4 that

$$
\operatorname{ext}_{p}^{i}(G, C)=\operatorname{ext}_{p}^{i}\left(C_{G}(g), 1\right)
$$

4.C. Subgroups, quotients. - The next results follows respectively from Corollaries 3.6, 3.7 and from Theorem 3.10:

Proposition 4.7. - Let $H$ be a subgroup of $G$ of index prime to $p$ and let $N$ be a normal subgroup of $G$. Then:

(a) $\ell_{p}(G, 1) \leqslant \ell_{p}(H, 1)$.

(b) If $H$ controls the fusion of $p$-elements, then $\ell_{p}(G, 1)=\ell_{p}(H, 1)$ and $\operatorname{ext}_{p}^{i}(G, 1)=\operatorname{ext}_{p}^{i}(H, 1)$ for every $i \geqslant 0$.

(c) $\ell_{p}(G / N, 1) \leqslant \ell_{p}(G, 1)$.

(d) If $|N|$ is prime to $p$, then $\ell_{p}(G, 1)=\ell_{p}(H, 1)$ and $\operatorname{ext}_{p}^{i}(G, 1)=\operatorname{ext}_{p}^{i}(H, 1)$ for every $i \geqslant 0$.

4.D. Direct products. - We study here the behaviour of the invariants $\ell_{p}(G, C)$ and $\operatorname{ext}_{p}^{1}(G, C)$ with respect to taking direct products. We first recall the following result on finite dimensional algebras:

Proposition 4.8. - Let $A$ and $B$ be two finite dimensional k-algebras. Then:

(a) $\operatorname{Rad}\left(A \otimes_{k} B\right)=A \otimes_{k}(\operatorname{Rad} B)+(\operatorname{Rad} A) \otimes_{k} B$.

(b) If $A / \operatorname{Rad} A \simeq k$ and $B / \operatorname{Rad} B \simeq k$, then

$$
\operatorname{Rad}\left(A \otimes_{k} B\right) / \operatorname{Rad}\left(A \otimes_{k} B\right)^{2} \simeq(\operatorname{Rad} A) /(\operatorname{Rad} A)^{2} \oplus(\operatorname{Rad} B) /(\operatorname{Rad} B)^{2} .
$$

Proof. - (a) is proved for instance in [CR, Proof of 10.39]. Let us now prove (b). Let $\theta$ : $(\operatorname{Rad} A) \oplus(\operatorname{Rad} B) \rightarrow \operatorname{Rad}\left(A \otimes_{k} B\right) / \operatorname{Rad}\left(A \otimes_{k} B\right)^{2}, a \oplus b \mapsto \overline{a \otimes_{k} 1+1 \otimes_{k} b}$. By (a), $\theta$ is surjective and $(\operatorname{Rad} A)^{2} \oplus(\operatorname{Rad} B)^{2}$ is contained in the kernel of $\theta$. Now the result follows from dimension reasons (using (a)).

Proposition 4.9. - Let $G$ and $H$ be two finite groups and let $C \in G_{p^{\prime}} / \sim$ and $D \in H_{p^{\prime}} / \sim$. Then

and

$$
\begin{gathered}
\ell_{p}(G \times H, C \times D)=\ell_{p}(G, C)+\ell_{p}(H, D)-1 \\
\operatorname{ext}_{p}^{1}(G \times H, C \times D)=\operatorname{ext}_{p}^{1}(G, C)+\operatorname{ext}_{p}^{1}(H, D) .
\end{gathered}
$$

Proof. - Write $A=k \mathcal{R}_{\mathfrak{p}}(G, C)$ and $B=k \mathcal{R}_{\mathfrak{p}}(H, D)$. It is easily checked that $k \mathcal{R}_{\mathfrak{p}}(G \times H, C \times$ $D)=A \otimes_{k} B$. So the first equality follows from Propositon 4.8 (a) and from the commutativity of $A$ and $B$. Moreover $A /(\operatorname{Rad} A) \simeq k$ and $B /(\operatorname{Rad} B) \simeq k$. In particular

$$
\operatorname{dim}_{k} \operatorname{Ext}_{A}^{1}(A / \operatorname{Rad} A, A / \operatorname{Rad} A)=\operatorname{dim}_{k}(\operatorname{Rad} A) /(\operatorname{Rad} A)^{2} .
$$

So the second equality follows from Proposition 4.8 (b). 
4.E. Abelian groups. - We compute here the invariants $\ell_{p}(G, 1)$ and $\operatorname{ext}_{p}^{1}(G, 1)$ whenever $G$ is abelian. If $G$ is abelian, then there is a (non-canonical) isomorphism of algebras $k \mathcal{R}(G) \simeq k G$.

Let us first start with the cyclic case:

$$
\text { if } G \text { is cyclic, then } \ell_{p}(G)=|G|_{p}+1 \text { and } \operatorname{ext}_{p}^{1}(G, 1)= \begin{cases}1 & \text { if } p \text { divides }|G|, \\ 0 & \text { otherwise. }\end{cases}
$$

Therefore, by Proposition 4.9 , we have: if $G_{1}, \ldots, G_{n}$ are cyclic, then

$$
\ell_{p}\left(G_{1} \times \cdots \times G_{n}\right)=\left|G_{1}\right|_{p}+\cdots+\left|G_{n}\right|_{p}-n+1 .
$$

and

$$
\operatorname{ext}_{p}^{1}\left(G_{1} \times \cdots \times G_{n}\right)=\mid\left\{1 \leqslant i \leqslant n \mid p \text { divides } G_{i}\right\} \mid \text {. }
$$

\section{The symmetric group}

In this section, and only in this section, we fix a non-zero natural number $n$ and a prime number $p$ and we assume that $G=\mathfrak{S}_{n}$, that $\mathcal{O}=\mathbb{Z}$ and that $\mathfrak{p}=p \mathbb{Z}$. Let $\mathbb{F}_{p}=k$. It is well-known that $\mathbb{Q}$ and $\mathbb{F}_{p}$ are splitting fields for $\mathfrak{S}_{n}$. For simplification, we set $\mathcal{R}_{n}=\mathcal{R}\left(\mathfrak{S}_{n}\right)$ and $\overline{\mathcal{R}}_{n}=\mathbb{F}_{p} \mathcal{R}\left(\mathfrak{S}_{n}\right)$. We investigate further the structure of $\overline{\mathcal{R}}_{n}$. This is a continuation of the work started in [B] in which the description of the descending Loewy series of $\overline{\mathcal{R}}_{n}$ was obtained.

We first introduce some notation. Let $\operatorname{Part}(n)$ denote the set of partitions of $n$. If $\lambda=$ $\left(\lambda_{1}, \ldots, \lambda_{r}\right) \in \operatorname{Part}(n)$ and if $1 \leqslant i \leqslant n$, we denote by $r_{i}(\lambda)$ the number of occurences of $i$ as a part of $\lambda$. We set

$$
\pi_{p}(\lambda)=\sum_{i=1}^{n}\left[\frac{r_{i}(\lambda)}{p}\right]
$$

where, for $x \in \mathbb{R}, x \geqslant 0$, we denote by $[x]$ the unique natural number $m \geqslant 0$ such that $m \leqslant x<$ $m+1$. Note that $\pi_{p}(\lambda) \in\{0,1,2, \ldots,[n / p]\}$ and recall that $\lambda$ is $p$-regular (resp. p-singular) if and only if $\pi_{p}(\lambda)=0$ (resp. $\pi_{p}(\lambda) \geqslant 1$ ). We denote by $\mathfrak{S}_{\lambda}$ the Young subgroup canonically isomorphic to $\mathfrak{S}_{\lambda_{1}} \times \cdots \times \mathfrak{S}_{\lambda_{r}}$, by $1_{\lambda}$ the trivial character of $\mathfrak{S}_{\lambda}$, and by $c_{\lambda}$ an element of $\mathfrak{S}_{\lambda}$ with only $r$ orbit in $\{1,2, \ldots, n\}$. Let $C_{\lambda}$ denote the conjugacy class of $c_{\lambda}$ in $\mathfrak{S}_{n}$. Then the map $\operatorname{Part}(n) \rightarrow \mathfrak{S}_{n} / \sim$, $\lambda \mapsto C_{\lambda}$ is a bijection. Let $W(\lambda)=N_{\mathfrak{S}_{n}}\left(\mathfrak{S}_{\lambda}\right) / \mathfrak{S}_{\lambda}$. Then

$$
W(\lambda) \simeq \prod_{i=1}^{n} \mathfrak{S}_{r_{i}(\lambda)}
$$

In particular, $\pi_{p}(\lambda)$ is the $p$-rank of $W(\lambda)$, where the $p$-rank of a finite group is the maximal rank of an elementary abelian subgroup. Now, we set $\varphi_{\lambda}=\operatorname{Ind}_{\mathfrak{S}_{\lambda}}^{\mathfrak{S}_{n}} 1_{\lambda}$. An old result of Frobenius says that

$$
\left(\varphi_{\lambda}\right)_{\lambda \in \operatorname{Part}(n)} \text { is a } \mathbb{Z} \text {-basis of } \mathcal{R}_{n}
$$

(see for instance [GP, Theorem 5.4.5 (b)]). Now, if $i \geqslant 1$, let

$$
\operatorname{Part}_{p}^{\geqslant i}(n)=\left\{\lambda \in \operatorname{Part}(n) \mid \pi_{p}(\lambda) \geqslant i\right\}
$$

and

$$
\operatorname{Part}_{p}^{i}(n)=\left\{\lambda \in \operatorname{Part}(n) \mid \pi_{p}(\lambda)=i\right\} .
$$

Then, by $[\mathbf{B}$, Theorem A], we have

$$
\left(\operatorname{Rad} \overline{\mathcal{R}}_{n}\right)^{i}=\underset{\lambda \in \operatorname{Part}_{p}^{(i)}(n)}{\oplus} \mathbb{F}_{p} \bar{\varphi}_{\lambda}
$$


Let $\operatorname{Part}_{p^{\prime}}(n)$ denote the set of partitions of $n$ whose parts are prime to $p$. Then the map $\operatorname{Part}_{p^{\prime}}(n) \rightarrow G_{p^{\prime}} / \sim, \lambda \mapsto C_{\lambda}$ is bijective. We denote by $\tau_{p^{\prime}}(\lambda)$ the unique partition of $n$ such that $\left(c_{\lambda}\right)_{p^{\prime}} \in C_{\tau_{p^{\prime}}(\lambda)}$. If $\lambda=\left(\lambda_{1}, \lambda_{2}, \ldots, \lambda_{r}\right)$, the partition $\tau_{p^{\prime}}(\lambda)$ is obtained as follows. Let

$$
\lambda^{\prime}=(\underbrace{\left(\lambda_{1}\right)_{p^{\prime}}, \ldots,\left(\lambda_{1}\right)_{p^{\prime}}}_{\left(\lambda_{1}\right)_{p} \text { times }}, \ldots, \underbrace{\left(\lambda_{r}\right)_{p^{\prime}}, \ldots,\left(\lambda_{r}\right)_{p^{\prime}}}_{\left(\lambda_{r}\right)_{p} \text { times }}) .
$$

Then $\tau_{p^{\prime}}(\lambda)$ is obtained from $\lambda^{\prime}$ by reordering the parts. The map $\tau_{p^{\prime}}: \operatorname{Part}(n) \rightarrow \operatorname{Part}_{p^{\prime}}(n)$ is obviously surjective. If $\lambda \in \operatorname{Part}_{p^{\prime}}(n)$, we set for simplification $\mathcal{R}_{n, p}(\lambda)=\mathcal{R}_{p \mathbb{Z}}\left(\mathfrak{S}_{n}, C_{\lambda}\right)$ and $\overline{\mathcal{R}}_{n}(\lambda)=\mathbb{F}_{p} \mathcal{R}_{p \mathbb{Z}}\left(\mathfrak{S}_{n}, C_{\lambda}\right)$. In other words,

$$
\mathbb{Z}_{p \mathbb{Z}} \mathcal{R}_{n}=\underset{\lambda \in \operatorname{Part}_{p^{\prime}}(n)}{\oplus} \mathcal{R}_{n, p}(\lambda)
$$

and

$$
\overline{\mathcal{R}}_{n}=\underset{\lambda \in \operatorname{Part}_{p^{\prime}}(n)}{\oplus} \overline{\mathcal{R}}_{n}(\lambda)
$$

are the decomposition of $\mathbb{Z}_{p \mathbb{Z}} \mathcal{R}_{n}$ and $\overline{\mathcal{R}}_{n}$ as a sum of blocks. We now make the result 5.3 more precise:

Proposition 5.4. - If $\lambda \in \operatorname{Part}_{p^{\prime}}(n)$ and if $i \geqslant 0$, then

$$
\operatorname{dim}_{\mathbb{F}_{p}}\left(\operatorname{Rad} \overline{\mathcal{R}}_{n}(\lambda)\right)^{i}=\left|\tau_{p^{\prime}}^{-1}(\lambda) \cap \operatorname{Part}_{p}^{\geqslant i}(n)\right| \text {. }
$$

Proof. - If $\lambda$ and $\mu$ are two partitions of $n$, we write $\lambda \subset \mu$ if $\mathfrak{S}_{\lambda}$ is conjugate to a subgroup of $\mathfrak{S}_{\mu}$. This defines an order on $\operatorname{Part}(n)$. On the other hand, if $d \in \mathfrak{S}_{n}$, we denote by $\lambda \cap{ }^{d} \mu$ the unique partition $\nu$ of $n$ such that $\mathfrak{S}_{\lambda} \cap{ }^{d} \mathfrak{S}_{\mu}$ is conjugate to $\mathfrak{S}_{\nu}$. Then, by the Mackey formula for tensor product (see for instance [CR, Theorem 10.18]), we have

$$
\varphi_{\lambda} \varphi_{\mu}=\sum_{d \in\left[\mathfrak{S}_{\lambda} \backslash \mathfrak{S}_{n} / \mathfrak{S}_{\mu}\right]} \varphi_{\lambda \cap{ }^{d} \mu}
$$

Here, $\left[\mathfrak{S}_{\lambda} \backslash \mathfrak{S}_{n} / \mathfrak{S}_{\mu}\right]$ denotes a set of representatives of the $\left(\mathfrak{S}_{\lambda}, \mathfrak{S}_{\mu}\right)$-double cosets in $\mathfrak{S}_{n}$. This shows that, if we fixe $\lambda_{0} \in \operatorname{Part}(n)$, then $\oplus_{\lambda \subset \lambda_{0}} \mathbb{Z} \varphi_{\lambda}$ and $\oplus_{\lambda \subsetneq \lambda_{0}} \mathbb{Z} \varphi_{\lambda}$ are sub- $\mathcal{R}(G)$-module of $\mathcal{R}(G)$. We denote by $\mathcal{D}_{\lambda}^{\mathbb{Z}}$ the quotient of these two modules. Then

$$
K \otimes_{\mathbb{Z}} \mathcal{D}_{\lambda}^{\mathbb{Z}} \simeq \mathcal{D}_{C_{\lambda}}
$$

This follows for instance from [GP, Proposition 2.4.4]. Consequently,

$$
k \otimes_{\mathbb{Z}} \mathcal{D}_{\lambda}^{\mathbb{Z}} \simeq \overline{\mathcal{D}}_{C_{\lambda}}
$$

It then follows from Proposition 2.14 that

$$
k \otimes_{\mathbb{Z}} \mathcal{D}_{\lambda}^{\mathbb{Z}} \simeq k \otimes_{\mathbb{Z}} \mathcal{D}_{\mu}^{\mathbb{Z}} \text { if and only if } \tau_{p^{\prime}}(\lambda)=\tau_{p^{\prime}}(\mu) .
$$

Now the Theorem follows from easily from (3), (4) and 5.3.

Now, if $\lambda \in \operatorname{Part}_{p^{\prime}}(n)$, then $C_{\mathfrak{S}_{n}}\left(w_{\lambda}\right)$ contains a normal $p^{\prime}$-subgroup $N_{\lambda}$ such that $C_{\mathfrak{S}_{n}}\left(w_{\lambda}\right) / N_{\lambda}$ is isomorphic to $W(\lambda)$. We denote by $1^{n}$ the partition $(1,1, \ldots, 1)$ of $n$. It follows from Theorem 3.4 and Theorem 3.10 that

$$
\mathcal{R}_{n, p}(\lambda) \simeq \mathcal{R}_{p \mathbb{Z}}(W(\lambda), 1) \simeq \bigotimes_{i=1}^{n} \mathcal{R}_{r_{i}(\lambda), p}\left(1^{r_{i}(\lambda)}\right)
$$

and

$$
\overline{\mathcal{R}}_{n}(\lambda) \simeq \overline{\mathcal{R}}(W(\lambda), 1) \simeq \bigotimes_{i=1}^{n} \overline{\mathcal{R}}_{r_{i}(\lambda)}\left(1^{r_{i}(\lambda)}\right) .
$$

We denote by $\log _{p} n$ the real number $x$ such that $p^{x}=n$. Then:

Corollary 5.7. - If $\lambda \in \operatorname{Part}_{p^{\prime}}(n)$, then

and

$$
\operatorname{ext}_{p}^{1}\left(\mathfrak{S}_{n}, C_{\lambda}\right)=\sum_{i=1}^{n}\left[\log _{p} r_{i}(\lambda)\right]
$$

$$
\ell_{p}\left(\mathfrak{S}_{n}, C_{\lambda}\right)=\pi_{p}(\lambda)+1 \text {. }
$$


Proof. — By 5.6 and by Proposition 4.9, both equalities need only to be proved whenever $\lambda=\left(1^{n}\right)$. So we assume that $\lambda=\left(1^{n}\right)$.

Let us show the first equality. By Proposition 5.4, we are reduced to show that $\mid \tau_{p^{\prime}}^{-1}\left(1^{n}\right) \cap$ $\operatorname{Part}_{p}^{1}(n) \mid=\left[\log _{p} n\right]$. Let $r=\left[\log _{p} n\right]$. In other words, we have $p^{r} \leqslant n<p^{r+1}$. If $1 \leqslant i \leqslant r$, write $n-p^{i}=\sum_{j=0}^{r} a_{i j} p^{j}$ with $0 \leqslant a_{i j}<p-1$ (the $a_{i j}$ 's are uniquely determined). Let

$$
\lambda(i)=(\underbrace{p^{r}, \ldots, p^{r}}_{a_{i r} \text { times }}, \ldots, \underbrace{p^{i}, \ldots, p^{i}}_{a_{i r} \text { times }} \underbrace{p^{i-1}, \ldots, p^{i-1}}_{\left(p+a_{i-1, r}\right) \text { times }}, \underbrace{p^{i-2}, \ldots, p^{i-2}}_{a_{i-2, r} \text { times }}, \ldots, \underbrace{1, \ldots, 1}_{a_{0 r} \text { times }}) .
$$

The result will follow from the following equality

$$
\tau_{p^{\prime}}^{-1}\left(1^{n}\right) \cap \operatorname{Part}_{p}^{1}(n)=\{\lambda(1), \lambda(2), \ldots, \lambda(r)\} .
$$

So let us now prove $(*)$. Let $I=\{\lambda(1), \lambda(2), \ldots, \lambda(r)\}$. It is clear that $I \subset \tau_{p^{\prime}}^{-1}\left(1^{n}\right) \cap \operatorname{Part}_{p}^{1}(n)$. Now, let $\lambda \in \tau_{p^{\prime}}^{-1}\left(1^{n}\right) \cap \operatorname{Part}_{p}^{1}(n)$. Then there exists a unique $i \in\{1,2, \ldots, r\}$ such that $r_{p^{i-1}}(\lambda) \geqslant p$. Moreover, $r_{p^{i-1}}(\lambda)<2 p$. So, if we set $r_{p^{j}}^{\prime}=r_{r_{j}}(\lambda)$ if $j \neq i-1$ and $r_{p^{i-1}}^{\prime}=r_{p^{i-1}}(\lambda)-p$, we get that $0 \leqslant r_{p^{j}}^{\prime} \leqslant p-1$ and $n-p^{i}=\sum_{j=0}^{r} r_{p^{j}}^{\prime} p^{j}$. This shows that $r_{p^{j}}^{\prime}=a_{i j}$, so $\lambda=\lambda(i)$.

Let us now show the second equality fo the Corollary. By Proposition 5.4, we only need to show that $\left|\tau_{p^{\prime}}^{-1}\left(1^{n}\right) \cap \operatorname{Part}_{p}^{[n / p]}(n)\right| \geqslant 1$. But in fact, it is clear that $\tau_{p^{\prime}}^{-1}\left(1^{n}\right) \cap \operatorname{Part}_{p}^{[n / p]}(n)=\left\{1^{n}\right\}$.

Corollary 5.8. - We have

and

$$
\begin{gathered}
\operatorname{dim}_{\mathbb{F}_{p}}\left(\operatorname{Rad} \overline{\mathcal{R}}_{n}\left(1^{n}\right)\right)^{[n / p]}=1 \\
\operatorname{dim}_{\mathbb{F}_{p}} \operatorname{Ext}_{\overline{\mathcal{R}}_{n}}\left(\overline{\mathcal{D}}_{1^{n}}, \overline{\mathcal{D}}_{1^{n}}\right)=\left[\log _{p} n\right] .
\end{gathered}
$$

In particular, $\ell_{p}\left(\mathfrak{S}_{n}, 1\right)=\ell_{p}\left(\mathfrak{S}_{n}\right)=[n / p]$.

Proof. - This is just a particular case of the previous corollary. The first equality has been obtained in the course of the proof of the previous corollary.

\section{Dihedral groups}

Let $n \geqslant 1$ and $m \geqslant 0$ be two natural numbers. We assume in this section, and only in this subsection, that $G=D_{2^{n}(2 m+1)}$ is the dihedral group of order $2^{n}(2 m+1)$ and that $p=2$.

Proposition 6.1. - If $n \geqslant 1$ and $m \geqslant 0$ are natural numbers, then

$$
\ell_{2}\left(D_{2^{n}(2 m+1)}, 1\right)= \begin{cases}2 & \text { if } n=1, \\ 3 & \text { if } n=2, \\ 2^{n-2}+1 & \text { if } n \geqslant 3 .\end{cases}
$$

and

$$
\operatorname{ext}_{2}^{1}\left(D_{2^{n}(2 m+1)}, 1\right)= \begin{cases}1 & \text { if } n=1 \\ 2 & \text { if } n=2 \\ 3 & \text { if } n \geqslant 3\end{cases}
$$

Proof. - Let $N$ be the normal subgroup of $G$ of order $2 m+1$. Then $G \simeq D_{2^{n}} \ltimes N$. So, by Proposition 4.7 (d), we may, and we will, assume that $m=0$. If $n=1$ or 2 the the result is easily checked. Therefore, we may, and we will, assume that $n \geqslant 3$.

Write $h=2^{n-1}$. We have

$$
G=<s, t \mid s^{2}=t^{2}=(s t)^{h}=1>.
$$

Let $H=<s t>$ and $S=\left\langle s>\right.$. Then $|H|=2^{n-1}=h$ and $G=S \ltimes H$. We fix a primitive $h$-th root of unity $\zeta \in \mathcal{O}^{\times}$. If $i \in \mathbb{Z}$, we denote by $\xi_{i}$ the unique linear character of $H$ such that $\xi_{i}(s t)=\zeta^{i}$. Then $\operatorname{Irr} H=\left\{\xi_{0}, \xi_{1}, \ldots, \xi_{h-1}\right\}$, and $\xi_{0}=1_{H}$. 
Since $n \geqslant 3, h$ is even and, if we write $h=2 h^{\prime}$, then $h^{\prime}=2^{n-2}$ is also even. For $i \in \mathbb{Z}$, we set

$$
\chi_{i}=\operatorname{Ind}_{H}^{G} \xi_{i} .
$$

It is readily seen that $\chi_{i}=\chi_{-i}$, that $\chi_{i+h}=\chi_{i}$ and that

$$
\chi_{i} \chi_{j}=\chi_{i+j}+\chi_{i-j} .
$$

Let $\varepsilon$ (resp. $\varepsilon_{s}$, resp. $\varepsilon_{t}$ ) be the unique linear character of order 2 such that $\varepsilon(s t)=1$ (resp. $\varepsilon_{s}(s)=1$, resp. $\left.\varepsilon_{t}(t)=1\right)$. Then

$$
\begin{aligned}
\chi_{0} & =1_{G}+\varepsilon, \\
\chi_{h^{\prime}} & =\varepsilon_{s}+\varepsilon_{t},
\end{aligned}
$$

and, if $h^{\prime}$ does not divide $i$,

$$
\chi_{i} \in \operatorname{Irr} G \text {. }
$$

Moreover, $|\operatorname{Irr} G|=h^{\prime}+3$ and

$$
\operatorname{Irr} G=\left\{1_{G}, \varepsilon, \varepsilon_{s}, \varepsilon_{t}, \chi_{1}, \chi_{2}, \ldots, \chi_{h^{\prime}-1}\right\} .
$$

Finally, note that

$$
\varepsilon_{s} \chi_{i}=\varepsilon_{t} \chi_{i}=\chi_{i+h^{\prime}}
$$

Let us start by finding a lower bound for $\ell_{2}(G)$. First, notice that the following equality holds: for all $i, j \in \mathbb{Z}$ and every $r \geqslant 0$, we have

$$
\left(\bar{\chi}_{i}+\bar{\chi}_{j}\right)^{2^{r}}=\bar{\chi}_{2^{r} i}+\bar{\chi}_{2^{r} j} .
$$

Proof of 6.4 . - Recall that $\bar{\chi}_{i}$ denotes the image of $\chi_{i}$ in $k \mathcal{R}(G)$. We proceed by induction on $r$. The case $r=0$ is trivial. The induction step is an immediate consequence of 6.2.

Note also the following fact (which follows from Example 2.18):

$$
\text { If } i \in \mathbb{Z} \text {, then } \bar{\chi}_{i} \in \operatorname{Rad} k \mathcal{R}(G) \text {. }
$$

Therefore,

$$
\ell_{2}(G) \geqslant 2^{n-2}+1 \text {. }
$$

Proof of 6.6. - By 6.4, we have immediately that $\left(\bar{\chi}_{0}+\bar{\chi}_{1}\right)^{2^{n-2}}=\bar{\chi}_{0}+\bar{\chi}_{h^{\prime}} \neq 0$ and, by $6.5, \bar{\chi}_{0}+\bar{\chi}_{1} \in \operatorname{Rad} k \mathcal{R}(G)$.

By Example 2.18, we have

$$
\left(\overline{1}_{G}+\bar{\varepsilon}_{s}, \bar{\chi}_{0}, \bar{\chi}_{1}, \ldots, \bar{\chi}_{h^{\prime}}\right) \text { is a } k \text {-basis of } \operatorname{Rad} k \mathcal{R}(G) .
$$

By 6.3 and 6.2, we get that

$$
\left(\bar{\chi}_{i}+\bar{\chi}_{i+2}\right)_{0 \leqslant i \leqslant h^{\prime}-2} \text { is a } k \text {-basis of }(\operatorname{Rad} k \mathcal{R}(G))^{2} .
$$

This shows that $\operatorname{ext}_{p}^{1}(G)=3$, as expected. It follows that, if $n \geqslant 3$ and $2 \leqslant i \leqslant 2^{n-2}+1$, then

$$
\operatorname{dim}_{k}\left(\operatorname{Rad} k \mathcal{R}\left(D_{2^{n}}\right)\right)^{i}=2^{n-2}+1-i
$$

Proof of 6.9. - Let $d_{i}=\operatorname{dim}_{k}\left(\operatorname{Rad} k \mathcal{R}\left(D_{2^{n}}\right)\right)^{i}$. By 6.8, we have $d_{2}=2^{n-2}-1$. By 6.6 , we have $d_{2^{n-2}} \geqslant 1$. Moreover, $d_{1}>d_{2}>d_{3}>\ldots$ So the proof of 6.9 is complete.

In particular, we get:

$$
\text { If } n \geqslant 3 \text {, then }\left(\operatorname{Rad} k \mathcal{R}\left(D_{2^{n}}\right)\right)^{2^{n-2}}=k\left(\overline{1}_{D_{2^{n}}}+\bar{\varepsilon}+\bar{\varepsilon}_{s}+\bar{\varepsilon}_{t}\right) .
$$

and $\ell_{2}\left(D_{2^{n}}\right)=2^{n-2}+1$, as expected. 


\section{Some tables}

For $0 \leqslant i \leqslant \ell_{p}(G)-1$, we set $d_{i}=\operatorname{dim}_{k}(\operatorname{Rad} k \mathcal{R}(G))^{i}$. Note that $d_{0}=|G / \sim|$ and $d_{0}-d_{1}=$ $\left|G_{p^{\prime}} / \sim\right|$. In this section, we give tables containing the values $\ell_{p}(G), \ell_{p}(G, 1), S_{p}(G), \operatorname{ext}_{p}^{1}(G, 1)$ and the sequence $\left(d_{0}, d_{1}, d_{2}, \ldots\right)$ for various groups. These computations have been made using GAP3 [GAP3].

These computations show that, if $G$ satisfies at least one of the following conditions:

(1) $|G| \leqslant 200$;

(2) $G$ is a subgroup of $\mathfrak{S}_{8}$;

(3) $G$ is one of the groups contained in the next tables;

then $\ell_{p}(G, 1)=\ell_{p}\left(N_{G}(P), 1\right)$ (here, $P$ denotes a Sylow $p$-subgroup of $G$ ). Note also that this equality holds if $P$ is abelian (see Example 3.8 ).

Question. Is it true that $\ell_{p}(G, 1)=\ell_{p}\left(N_{G}(P), 1\right)$ ?

The first table contains the datas for the the exceptional Weyl groups, the second table is for the alternating groups $\mathfrak{A}_{n}$ for $5 \leqslant n \leqslant 12$, the third table is for some small finite simple groups, and the last table is for the groups $\operatorname{PSL}(2, q)$ for $q$ a prime power $\leqslant 27$.

\begin{tabular}{|c|c|c||c|c|l|c|c|}
\hline$G$ & $|G|$ & $p$ & $\ell_{p}(G)$ & $S_{p}(G)$ & $d_{0}, d_{1}, d_{2}, \ldots$ & $\ell_{p}(G, 1)$ & ext $_{p}^{1}(G, 1)$ \\
\hline \hline$W\left(E_{6}\right)$ & 51840 & 2 & 5 & 10 & $25,19,9,3,1$ & 5 & 3 \\
& $2^{7} .3^{4} .5$ & 3 & 4 & 5 & $25,13,4,1$ & 4 & 2 \\
& & 5 & 2 & 2 & 25,2 & 2 & 1 \\
\hline$W\left(E_{7}\right)$ & 2903040 & 2 & 7 & 24 & $60,52,35,18,7,3,1$ & 7 & 4 \\
& $2^{10} .3^{4} .5 .7$ & 3 & 4 & 5 & $60,30,8,2$ & 4 & 2 \\
& & 5 & 2 & 2 & 60,6 & 2 & 1 \\
& & 7 & 2 & 2 & 60,2 & 2 & 1 \\
\hline$W\left(E_{8}\right)$ & 696729600 & 2 & 8 & 32 & $112,100,68,36,17,7,3,1$ & 8 & 5 \\
& $2^{14} .3^{5} .5^{2} .7$ & 3 & 5 & 8 & $112,65,24,7,2$ & 5 & 2 \\
& & 5 & 3 & 3 & $112,17,2$ & 3 & 1 \\
& & 7 & 2 & 2 & 112,4 & 2 & 1 \\
\hline$W\left(F_{4}\right)$ & 1152 & 2 & 5 & 14 & $25,21,12,4,1$ & 5 & 4 \\
& $2^{7} .3^{2}$ & 3 & 3 & 4 & $25,11,2$ & 3 & 2 \\
\hline$W\left(H_{3}\right)$ & 120 & 2 & 3 & 4 & $10,6,1$ & 3 & 2 \\
& $2^{3} .3 .5$ & 3 & 2 & 2 & 10,2 & 2 & 1 \\
& & 5 & 3 & 3 & $10,4,2$ & 3 & 1 \\
\hline$W\left(H_{4}\right)$ & 14400 & 2 & 4 & 7 & $34,24,9,1$ & 3 & 3 \\
& $2^{6} .3^{2} .5^{2}$ & 3 & 3 & 3 & $34,11,2$ & 5 & 2 \\
& & 5 & 5 & 6 & $34,20,11,4,2$ & & 2 \\
\hline
\end{tabular}




\begin{tabular}{|c|c|c||c|c|l|c|c|}
\hline$G$ & $|G|$ & $p$ & $\ell_{p}(G)$ & $S_{p}(G)$ & $d_{0}, d_{1}, d_{2}, \ldots$ & $\ell_{p}(G, 1)$ & $\operatorname{ext}_{p}^{1}(G, 1)$ \\
\hline \hline $\mathfrak{A}_{5}$ & 60 & 2 & 2 & 2 & 5,1 & 2 & 1 \\
& $2^{2} .3 .5$ & 3 & 2 & 2 & 5,1 & 2 & 1 \\
& & 5 & 3 & 3 & $5,2,1$ & 3 & 1 \\
\hline $\mathfrak{A}_{6}$ & 360 & 2 & 3 & 3 & $7,2,1$ & 3 & 1 \\
& $2^{3} .3^{2} .5$ & 3 & 3 & 3 & $7,2,1$ & 3 & 1 \\
& & 5 & 3 & 3 & $7,2,1$ & 3 & 1 \\
\hline $\mathfrak{A}_{7}$ & 2520 & 2 & 3 & 3 & $9,3,1$ & 3 & 1 \\
& $2^{3} .3^{2} .5 .7$ & 3 & 3 & 3 & $9,3,1$ & 3 & 1 \\
& & 5 & 2 & 2 & 9,1 & 2 & 1 \\
& & 7 & 3 & 3 & $9,2,1$ & 3 & 1 \\
\hline $\mathfrak{A}_{8}$ & 20160 & 2 & 4 & 5 & $14,6,2,1$ & 4 & 2 \\
& $2^{6} .3^{2} .5 .7$ & 3 & 3 & 3 & $14,6,2$ & 3 & 1 \\
& & 5 & 3 & 3 & $14,3,1$ & 2 & 1 \\
& & 7 & 3 & 3 & $14,2,1$ & 3 & 1 \\
\hline $\mathfrak{A}_{9}$ & 181440 & 2 & 4 & 5 & $18,8,3,1$ & 4 & 2 \\
& $2^{6} .3^{4} .5 .7$ & 3 & 4 & 6 & $18,10,3,1$ & 4 & 3 \\
& & 5 & 3 & 3 & $18,4,1$ & 2 & 1 \\
& & 7 & 2 & 2 & 18,1 & 2 & 1 \\
\hline $\mathfrak{A}_{10}$ & 1814400 & 2 & 5 & 7 & $24,12,6,2,1$ & 5 & 2 \\
& $2^{7} .3^{4} .5^{2} .7$ & 3 & 4 & 6 & $24,13,4,1$ & 4 & 3 \\
& & 5 & 3 & 3 & $24,4,1$ & 3 & 1 \\
& & 7 & 3 & 3 & $24,3,1$ & 2 & 1 \\
\hline $\mathfrak{A}_{11}$ & 19958400 & 2 & 5 & 7 & $31,17,8,3,1$ & 5 & 2 \\
& $2^{7} .3^{4} .5^{2} .7 .11$ & 3 & 4 & 5 & $31,16,6,1$ & 4 & 2 \\
& & 5 & 3 & 3 & $31,6,1$ & 3 & 1 \\
& & 7 & 3 & 3 & $31,4,1$ & 2 & 1 \\
& & 11 & 3 & 3 & $31,2,1$ & 3 & 1 \\
\hline $\mathfrak{A}_{12}$ & 239500800 & 2 & 6 & 10 & $43,25,13,6,2,1$ & 6 & 2 \\
& $2^{9} .3^{5} .5^{2} .7 .11$ & 3 & 5 & 8 & $43,22,9,2,1$ & 5 & 3 \\
& & 5 & 3 & 3 & $43,10,2$ & 3 & 1 \\
& & 7 & 3 & 3 & $43,5,1$ & 2 & 1 \\
& & 11 & 3 & 3 & $43,2,1$ & 3 & 1 \\
\hline
\end{tabular}




\begin{tabular}{|c|c|c|c|c|c|c|c|}
\hline$G$ & $|G|$ & $p$ & $\ell_{p}(G)$ & $S_{p}(G)$ & $d_{0}, d_{1}, d_{2}, \ldots$ & $\ell_{p}(G, 1)$ & $\operatorname{ext}_{p}^{1}(G, 1)$ \\
\hline \multirow[t]{3}{*}{$\overline{G G L(3,2)}$} & 168 & 2 & 3 & 3 & $6,2,1$ & 3 & 1 \\
\hline & $2^{3} \cdot 3.7$ & 3 & 2 & 2 & 6,1 & 2 & 1 \\
\hline & & 7 & 3 & 3 & $6,2,1$ & 3 & 1 \\
\hline \multirow[t]{3}{*}{$S L(2,8)$} & 504 & 2 & 2 & 2 & 9,1 & 2 & 1 \\
\hline & $2^{3} \cdot 3^{2} \cdot 7$ & 3 & 5 & 5 & $9,4,3,2,1$ & 5 & 1 \\
\hline & & 7 & 4 & 4 & $9,3,2,1$ & 4 & 1 \\
\hline \multirow[t]{3}{*}{$S L(3,3)$} & 5616 & 2 & 5 & 5 & $12,5,3,2,1$ & 5 & 1 \\
\hline & $2^{4} .3^{3} .13$ & 3 & 3 & 3 & $12,3,1$ & 3 & 1 \\
\hline & & 13 & 5 & 5 & $12,4,3,2,1$ & 5 & 1 \\
\hline \multirow[t]{3}{*}{$S U(3,3)$} & 6048 & 2 & 6 & 7 & $14,9,6,4,2,1$ & 6 & 2 \\
\hline & $2^{5} \cdot 3^{3} \cdot 7$ & 3 & 3 & 3 & $14,5,1$ & 3 & 1 \\
\hline & & 7 & 3 & 3 & $14,2,1$ & 3 & 1 \\
\hline \multirow[t]{4}{*}{$M_{11}$} & 7920 & 2 & 5 & 5 & $10,5,3,2,1$ & 5 & 1 \\
\hline & $2^{4} \cdot 3^{2} \cdot 5.11$ & 3 & 2 & 2 & 10,2 & 2 & 1 \\
\hline & & 5 & 2 & 2 & 10,1 & 2 & 1 \\
\hline & & 11 & 3 & 3 & $10,2,1$ & 3 & 1 \\
\hline \multirow[t]{3}{*}{$P S p(4,3)$} & 25920 & 2 & 4 & 5 & $20,12,5,1$ & 4 & 2 \\
\hline & $2^{6} .3^{4} .5$ & 3 & 5 & 7 & $20,14,8,3,1$ & 5 & 2 \\
\hline & & 5 & 2 & 2 & 20,1 & 2 & 1 \\
\hline \multirow[t]{4}{*}{$M_{12}$} & 95040 & 2 & 4 & 7 & $15,9,3,1$ & 4 & 3 \\
\hline & $2^{6} \cdot 3^{3} \cdot 5 \cdot 11$ & 3 & 3 & 3 & $15,4,1$ & 3 & 1 \\
\hline & & 5 & 2 & 2 & 15,2 & 2 & 1 \\
\hline & & 11 & 3 & 3 & $15,2,1$ & 3 & 1 \\
\hline \multirow[t]{6}{*}{$J_{1}$} & 175560 & 2 & 2 & 2 & 15,4 & 2 & 1 \\
\hline & $2^{3} .3 .5 .7 .11 .19$ & 3 & 2 & 2 & 15,4 & 2 & 1 \\
\hline & & 5 & 3 & 3 & $15,6,3$ & 3 & 1 \\
\hline & & 7 & 2 & 2 & 15,1 & 2 & 1 \\
\hline & & 11 & 2 & 2 & 15,1 & 2 & 1 \\
\hline & & 19 & 4 & 4 & $15,3,2,1$ & 4 & 1 \\
\hline \multirow[t]{5}{*}{$M_{22}$} & 443520 & 2 & 4 & 5 & $12,5,2,1$ & 4 & 2 \\
\hline & $2^{7} .3^{2} \cdot 5.7 .11$ & 3 & 2 & 2 & 12,2 & 2 & 1 \\
\hline & & 5 & 2 & 2 & 12,1 & 2 & 1 \\
\hline & & 7 & 3 & 3 & $12,2,1$ & 3 & 1 \\
\hline & & 11 & 3 & 3 & $12,2,1$ & 3 & 1 \\
\hline \multirow[t]{4}{*}{$J_{2}$} & 604800 & 2 & 4 & 5 & $21,11,3,1$ & 4 & 2 \\
\hline & $2^{7} \cdot 3^{3} \cdot 5^{2} \cdot 7$ & 3 & 3 & 3 & $21,7,1$ & 3 & 1 \\
\hline & & 5 & 5 & 5 & $21,10,6,2,1$ & 5 & 1 \\
\hline & & 7 & 2 & 2 & 21,1 & 2 & 1 \\
\hline \multirow[t]{5}{*}{$H S$} & 44352000 & 2 & 5 & 9 & $24,15,8,3,1$ & 5 & 3 \\
\hline & $2^{9} \cdot 3^{2} \cdot 5^{3} \cdot 7.11$ & 3 & 2 & 2 & 24,5 & 2 & 1 \\
\hline & & 5 & 3 & 4 & $24,8,2$ & 3 & 2 \\
\hline & & 7 & 2 & 2 & 24,1 & 2 & 1 \\
\hline & & 11 & 3 & 3 & $24,2,1$ & 3 & 1 \\
\hline
\end{tabular}




\begin{tabular}{|c|c|c|c|c|c|c|c|}
\hline$G$ & $|G|$ & $p$ & $\ell_{p}(G)$ & $S_{p}(G)$ & $d_{0}, d_{1}, d_{2}, \ldots$ & $\ell_{p}(G, 1)$ & $\operatorname{ext}_{p}^{1}(G, 1)$ \\
\hline$P S L(2,2)$ & 6 & 2 & 2 & 2 & 3,1 & 2 & 1 \\
\hline$\simeq \mathfrak{S}_{3}$ & 2.3 & 3 & 2 & 2 & 3,1 & 2 & 1 \\
\hline$P S L(2,3)$ & 12 & 2 & 2 & 2 & 4,1 & 2 & 1 \\
\hline$\simeq \mathfrak{A}_{4}$ & $2^{2} .3$ & 3 & 3 & 3 & $4,2,1$ & 3 & 1 \\
\hline$P S L(2,4)$ & 60 & 2 & 2 & 2 & 5,1 & 2 & 1 \\
\hline$\simeq P S L(2,5)$ & $2^{2} .3 .5$ & 3 & 2 & 2 & 5,1 & 2 & 1 \\
\hline$\simeq \mathfrak{A}_{5}$ & & 5 & 3 & 3 & $5,2,1$ & 3 & 1 \\
\hline \multirow[t]{3}{*}{$P S L(2,7)$} & 168 & 2 & 3 & 3 & $6,2,1$ & 3 & 1 \\
\hline & $2^{3} .3 .7$ & 3 & 2 & 2 & 6,1 & 2 & 1 \\
\hline & & 7 & 3 & 3 & $6,2,1$ & 3 & 1 \\
\hline \multirow[t]{3}{*}{$P S L(2,8)$} & 504 & 2 & 2 & 2 & 9,1 & 2 & 1 \\
\hline & $2^{3} \cdot 3^{2} .7$ & 3 & 5 & 5 & $9,4,3,2,1$ & 5 & 1 \\
\hline & & 7 & 4 & 4 & $9,3,2,1$ & 4 & 1 \\
\hline \multirow{3}{*}{$\begin{array}{l}P S L(2,9) \\
\quad \simeq \mathfrak{A}_{6}\end{array}$} & 360 & 2 & 3 & 3 & $7,2,1$ & 3 & 1 \\
\hline & $2^{3} .3^{2} .5$ & 3 & 3 & 3 & $7,2,1$ & 3 & 1 \\
\hline & & 5 & 3 & 3 & $7,2,1$ & 3 & 1 \\
\hline \multirow[t]{4}{*}{$P S L(2,11)$} & 660 & 2 & 2 & 2 & 8,2 & 2 & 1 \\
\hline & $2^{2} .3 .5 .11$ & 3 & 2 & 2 & 8,2 & 2 & 1 \\
\hline & & 5 & 3 & 3 & $8,2,1$ & 3 & 1 \\
\hline & & 11 & 3 & 3 & $8,2,1$ & 3 & 1 \\
\hline \multirow[t]{4}{*}{$P S L(2,13)$} & 1092 & 2 & 2 & 2 & 9,2 & 2 & 1 \\
\hline & $2^{2} .3 .7 .13$ & 3 & 2 & 2 & 9,2 & 2 & 1 \\
\hline & & 7 & 4 & 4 & $9,3,2,1$ & 4 & 1 \\
\hline & & 13 & 3 & 3 & $9,2,1$ & 3 & 1 \\
\hline \multirow[t]{4}{*}{$P S L(2,16)$} & 4080 & 2 & 2 & 2 & 17,1 & 2 & 1 \\
\hline & $2^{4} .3 .5 .17$ & 3 & 3 & 3 & $17,5,2$ & 2 & 1 \\
\hline & & 5 & 5 & 5 & $17,6,4,2,1$ & 3 & 1 \\
\hline & & 17 & 9 & 9 & $17,8,7,6,5,4,3,2,1$ & 9 & 1 \\
\hline \multirow[t]{3}{*}{$P S L(2,17)$} & 2448 & 2 & 5 & 5 & $11,4,3,2,1$ & 5 & 1 \\
\hline & $2^{4} .3^{2} .17$ & 3 & 5 & 5 & $11,4,3,2,1$ & 5 & 1 \\
\hline & & 17 & 3 & 3 & $11,2,1$ & 3 & 1 \\
\hline \multirow[t]{4}{*}{$P S L(2,19)$} & 3420 & 2 & 2 & 2 & 12,3 & 2 & 1 \\
\hline & $2^{2} \cdot 3^{2} \cdot 5 \cdot 19$ & 3 & 5 & 5 & $12,4,3,2,1$ & 5 & 1 \\
\hline & & 5 & 3 & 3 & $12,4,2$ & 3 & 1 \\
\hline & & 19 & 3 & 3 & $12,2,1$ & 3 & 1 \\
\hline \multirow[t]{4}{*}{$P S L(2,23)$} & 6072 & 2 & 4 & 4 & $14,5,3,1$ & 3 & 1 \\
\hline & $2^{3} .3 .11 .23$ & 3 & 3 & 3 & $14,4,1$ & 2 & 1 \\
\hline & & 11 & 6 & 6 & $14,5,4,3,2,1$ & 6 & 1 \\
\hline & & 23 & 3 & 3 & $14,2,1$ & 3 & 1 \\
\hline \multirow[t]{4}{*}{$P S L(2,25)$} & 7800 & 2 & 4 & 4 & $15,5,3,1$ & 3 & 1 \\
\hline & $2^{3} \cdot 3.5^{2} \cdot 13$ & 3 & 3 & 3 & $15,4,1$ & 2 & 1 \\
\hline & & 5 & 3 & 3 & $15,2,1$ & 3 & 1 \\
\hline & & 13 & 7 & 7 & $15,6,5,4,3,2,1$ & 7 & 1 \\
\hline \multirow[t]{4}{*}{$P S L(2,27)$} & 9828 & 2 & 2 & 2 & 16,4 & 2 & 1 \\
\hline & $2^{2} \cdot 3^{3} \cdot 7.13$ & 3 & 3 & 3 & $16,2,1$ & 3 & 1 \\
\hline & & 7 & 4 & 4 & $16,6,4,2$ & 4 & 1 \\
\hline & & 13 & 7 & 7 & $16,6,5,4,3,2,1$ & 7 & 1 \\
\hline
\end{tabular}




\section{References}

[B] C. Bonnafé, A note on the Grothendieck ring of the symmetric group, C. R. Math. Acad. Sci. Paris, 342 (2006), no. 8, 533-538.

[CR] C.W. Curtis \& I. Reiner, Methods of representation thoery, vol. I, Wiley, New York, 1981; reprinted 1990 as Wiley Classics Library.

[D] M. Deiml, Zur Darstellungstheorie von Darstellungsringen, Ph.D. Thesis (Jena, 1997).

[DM] F. Digne \& J. Michel, Representations of finite groups of Lie type, London math. soc. student texts 21, Cambridge university press, 1991.

[GAP3] MARtin SchÖNERT ET. AL., GAP - Groups, Algorithms, and Programming - version 3 release 4 patchlevel 4. Lehrstuhl D für Mathematik, Rheinisch Westfälische Technische Hochschule, Aachen, Germany, 1997.

[GP] M. GECK \& G. PfeIfFer, Characters of finite Coxeter groups and Iwahori-Hecke algebras, London Math. Soc. Monographs, New series no. 21, Oxford University Press, 2000.

[H] G. Higman, The units of group-rings, Proc. London Math. Soc. 46 (1940), 231-248.

[I] M.I. IsAacs, Character theory of finite groups, Pure and Applied Mathematics 69, Academic Press, New York-London, 1976.

[L] G. Lusztig, Leading coefficients of character values of Hecke algebras, The Arcata Conference on Representations of Finite Groups (Arcata, Calif., 1986), 235-262, Proc. Sympos. Pure Math. 47, Part 2, Amer. Math. Soc., Providence, RI, 1987.

[S] A.I. SAksonov, The integral ring of characters of a finite group (Russian), Vesci Akad. Navuk BSSR Ser. Fiz.-Mat. Navuk 1966 (1966) no. 3, 69-76.

November 8, 2006

CÉDric Bonnafé, CNRS (UMR 6623), Laboratoire de Mathématiques de Besançon, Université de FrancheComté, 16 Route de Gray, 25030 Besançon Cedex, France • E-mail : bonnafe@math.univ-fcomte.fr Url : http://www-math.univ-f comte.fr/pp_Annu/CBONNAFE/ 